\title{
DEVELOPMENT OF GROUND-WATER RESOURCES \\ IN ORANGE COUNTY, TEXAS, AND ADJACENT \\ AREAS IN TEXAS AND LOUISIANA, 1971-80
}

By C.W. Bonnet and R.K. Gabrysch

U.S. GEO!OGICAL SURVEY

OPEN-FILE REPORT $82-330$

Prepared in cooperation with the Orange County

Commissioner's Court, the Sabine River Authority of rexas, and the Texas Department of Water Resources

Austin, Texas 
UNITED STATES DEPARTMENT OF THE INTERIOR

JAMES G. WATT, Secretary

GEOLOGICAL SURVEY

Dallas L. Peck, Director

For additional information write to:

District Chief

U.S. Geological Survey

649 Federal Building

$300 \mathrm{E}$. Eighth Street

Austin, TX 78701
For sale by:

Open-File Services Section

Branch of Distribution

U.S. Geological Survey, MS 306 Box 25425, Denver Federal Center Denver, C0 80225 


\section{CONTENTS}

Page

Abstract-1

Introduction-

Location and extent of the area-..... 2

Purpose and scope of the study

Previous investigations-_.

Acknowl edgments-_.

Well-numbering system-_... 5

Hydrologic and geologic units-_. 5

Ground-water development-_. 6

Pumpage-.......... 6

Changes in water levels

Subsidence-1..- 10

Water-qual ity changes-........... 10

Ground-water problems and future development_...

Summary-...... 19

Selected references-_-_. 20

Supplemental information 


\section{ILLUSTRATIONS}

Page

Figure 1. Index map showing location of study area--1..-..- 3

2. Map showing location of wells in the study area--...-...-.-. 4

3. Hydrographs of water levels in wells screened in the lower unit of the Chicot aquifer in Orange County,

4-9. Maps showing:

Texas, and Calcasieu Parish, Louisiana--.-.-.-.- 7

4. Approximate altitude of water levels in wells screened in the lower unit of the Chicot aquifer, spring 1980-.-.-.- 8

5. Changes in water levels in wells screened in the lower unit of the Chicot aquifer between spring 1971 and spring 1980--.--11

6. Location of bench marks and land-surface subsidence in the Beaumont, Orange, Port Arthur, Texas, area, 1953-55 to 1973----.--.-- 12

7. Land-surface subsidence in the Spindletop Dome area,

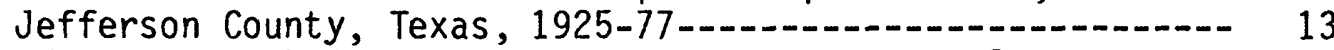

8. Land-surface subsidence in the Port Acres gas field area, Jefferson County, Texas, 1959-77-_...................... 14

9. Concentrations of chloride in water from wells screened in the lower unit of the Chicot aquifer at site $A$ in

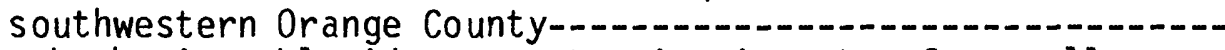

10. Graph showing chloride concentration in water from wells screened in the lower unit of the Chicot aquifer in southwestern Orange County-

11. Maps showing concentrations of chloride in water from wells screened in the lower unit of the Chicot aquifer at site $B$ in southeastern Orange County-...-

\section{TABLES}

Table 1. Pumpage of ground water from the lower unit of the Chicot aquifer in Orange County, 1963-79-1 9

2. Water levels in observation wells in the study area, 1975-80-......... 22

3. Records of selected wells in the study area, 1968-80-......- 32

4. Drillers' logs of selected wells in the study area, 1968-80-1-.-- 34

5. Chemical analyses of water from wells in Orange County, 1975-79-_ 


\section{METRIC CONVERSIONS}

For those readers interested in using the metric system, the metric equivalents of "inch-pound" units are given in parentheses. Factors for converting "inch-pound" units to metric equivalents are given in the following table:

\begin{tabular}{lcl}
\hline \multicolumn{1}{c}{ From } & Multiply by & \multicolumn{1}{c}{ To obtain } \\
\hline acre-foot & 0.001233 & cubic hectometer $\left(\mathrm{hm}^{3}\right)$ \\
foot & 0.3048 & meter $(\mathrm{m})$ \\
gallon per minute (gal/min) & 0.06309 & liter per second $(\mathrm{L} / \mathrm{s})$ \\
inch & 25.4 & millimeter $(\mathrm{mm})$ \\
mile & 1.609 & kilometer $(\mathrm{km})$ \\
million gallons per day & 0.04381 & cubic meter per second $\left(\mathrm{m}^{3} / \mathrm{s}\right)$ \\
(Mgal/d) & & \\
\hline
\end{tabular}

National Geodetic Vertical Datum of 1929 (NGVD of 1929): A geodetic datum derived from a general adjustment of the first-order level nets of both the United States and Canada, formerly called "mean sea level." 
DEVELOPMENT OF GROUND-WATER RESOURCES IN

ORANGE COUNTY, TEXAS, AND ADJACENT AREAS

IN TEXAS AND LOUISIANA, 1971-80

By

C. W. Bonnet and R. K. Gabrysch

\section{ABSTRACT}

Pumpage in Orange County from the lower unit of the Chicot aquifer averaged 21.2 million gallons per day ( 0.93 cubic meter per second) and pumpage from the upper unit of the Chicot averaged about 2 million gallons per day (0.088 cubic meter per second) from 1971-79. Annual pumpage increased in municipal areas and decreased in industrial areas with little net change in total annual pumpage during the report period. The major water-bearing unit in the study area is the Chicot aquifer, which overlies the Evangeline aquifer. The Evangeline aquifer is undeveloped in Orange County, but is developed at Evadale in Jasper County, Texas, and at Silsbee in Hardin County, Texas. Both aquifers consist of unconsolidated and discontinuous layers of sand and clay that gently dip toward the Gulf of Mexico.

Water levels in Orange County tended to stabilize or rise in areas where ground-water withdrawals decreased. El sewhere in Orange County, most water levels continued to decline, generally at a slower rate than before 1971 . Bench-mark elevations determined during 1973 show regional land-surface subsidence from 1918-73, generally attributed to ground-water development, to be less than 0.5 foot ( 0.15 meter). Locally, subsidence due to production of $0 i 1$, gas, saltwater, or sulfur was about 15 feet ( 4.6 meters) at Spindletop Dome, Jefferson County, Texas, and as much as 3 feet ( 1.0 meter) near Port Acres gas field, Jefferson County, Texas.

Although saltwater encroachment is evident in parts of southern Orange County, the encroachment is not expected to be detrimental if the ground-water pumping remains stable and the projected increase in demands for water is met with surface-water supplies. 


\section{INTRODUCTION \\ Location and Extent of the Area}

The study area, as described in this report, includes Orange County, southern Jasper County, southern Newton County, eastern Jefferson County, and southeastern Hardin County, Texas, and western Calcasieu and Cameron Parishes, Louisiana. The location of the study area is shown in figure 1.

\section{Purpose and Scope of the Study}

A program of continuing ground-water studies in Orange County, Texas, and adjacent areas in Texas and Louisiana in cooperation with the Texas Department of Water Resources and the Sabine River Authority of Texas began in March 1967 due to the need for systematic monitoring and appraisal of the changing groundwater conditions. Water levels were declining, and saltwater encroachment was suspected with a possibility of land-surface subsidence. In 1979, local cooperation was assumed by the Orange County Commissioner's Court. The continuing program includes the following items of work:

1. An inventory of all new large-capacity wells and the compilation of drillers' logs.

2. The establishment and maintenance of a network of observation wells for monitoring changes in water levels and changes in chemical quality, especially chlorides.

3. An annual inventory of pumpage for municipal supply and industrial use.

4. Determination of the extent of land-surface subsidence in Orange County.

5. The correlation of current data with previously collected data.

This report presents data collected since 1971, except for some of the data that were given in the 1971-74 progress report by Bonnet (1975), together with an analysis of the data. The location of wells for which data are included is shown in figure 2 .

\section{Previous Investigations}

The geology and ground-water resources of Orange County and adjacent areas are described in reports by Baker (1964) on the ground-water resources of Hardin County, Texas; Bonnet (1975) on ground-water data for Orange County and vicinity; Harder (1960) on the geology and ground-water resources of Calcasieu Parish, Louisiana; Jones and others $(1954,1956)$ on the geology of ground-water resources of southwestern Louisiana; McAdoo (1968, 1969, 1970) on ground-water data for Orange County and vicinity; Wesselman, on ground-water resources of Orange County (1965), Jasper and Newton Counties (1967), and Chambers and Jefferson Counties, Texas (1971); Zack (1971) updated the hydrologic studies in southwestern Louisiana; and Gabrysch and McAdoo (1972) on development of ground-water resources in the Orange County area, Texas and Louisiana, 1963-71.

\section{Acknowledgments}

The authors gratefully acknowledge the cooperation of the many land owners and industrial and city officials who provided data and granted access to water wells. The assistance of Mr. Albert Gray, Sabine River Authority of Texas, also is gratefully acknowledged. Others who contributed measurably to 


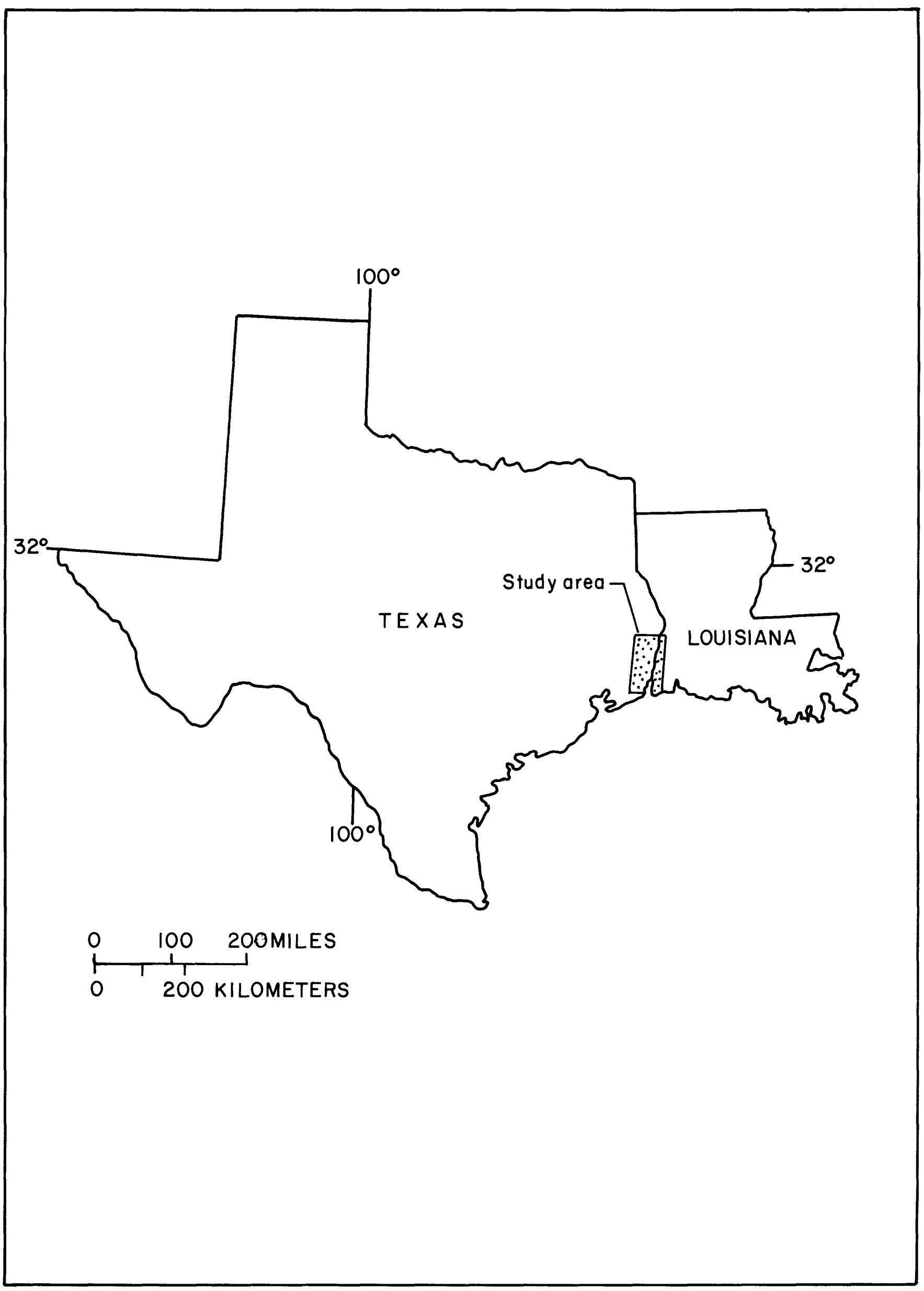

Figure 1.-Location of study area 


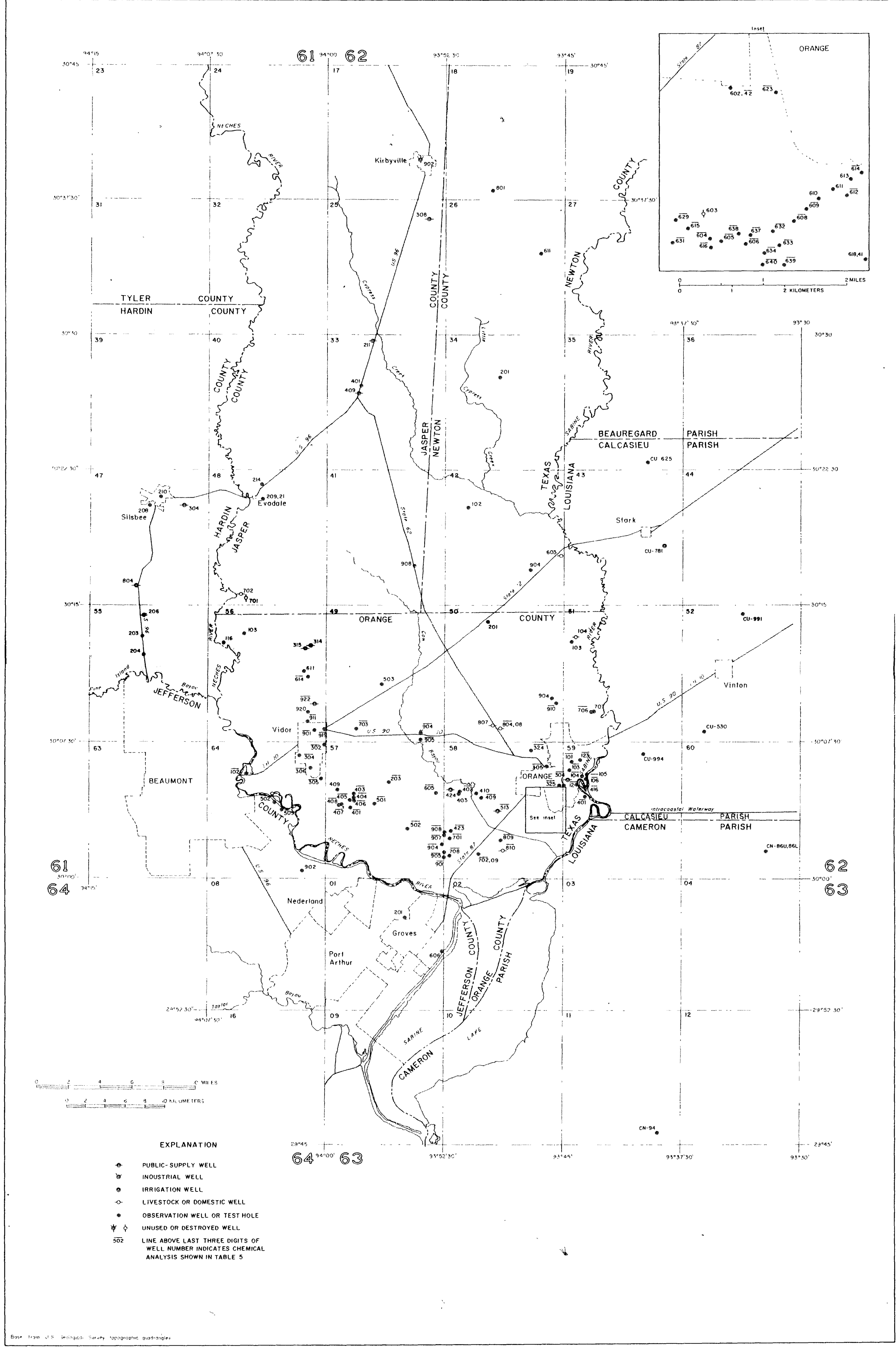


this report were: Mr. Carl Vincent and Mr. Bodie Pryor, Gulf Chemical Co.; Mr. R. A. Ogden and Mr. A. J. White, Firestone Petrochemical Center; Mr. E. L. Faulk, Mr. Jerry Black, and Mr. G. D. McWright, E. I. DuPont, Inc.; Mr. Cl ark Shupp, B. F. Goodrich Co.; Mr. Clarence Kite, Phillips Chemical Co.; and Mr. Louis Stough, Gulf States Utilities, Sabine Station.

\section{Well-Numbering System}

The well-numbering system in Texas was developed by the Texas Department of Water Resources for use throughout the State. Under this system, each 1degree quadrangle is given a number consisting of two digits. These are the first two digits in the well number. Each 1 -degree quadrangle is divided into 7-1/2-minute quadrangles which are given a two-digit number from 01 to 64 . These are the third and fourth digits of the well number. Each 7-1/2-minute quadrangle is divided into 2-1/2-minute quadrangles which are given a singledigit number from 1 to 9 . This is the fifth digit of the well number. Finally, each well within a 2-1/2-minute quadrangle is given a 2-digit number in the order in which it was inventoried, starting with 01 . These are the last two digits of the well number.

Only the last three digits of the well number are shown at each well location (fig. 2); the second two digits are shown in the northwest corner of each 7-1/2-minute quadrangle; and the first two digits are shown by the large block numerals in each 1 -degree quadrangle.

In addition to the seven-digit well number, a two-letter prefix is used to identify the county. The prefixes for Orange County and adjacent counties are as follows: Orange, UJ; Jasper, PR; Jefferson, PT; Hardin, LH; and Newton, TZ.

Wells inventoried in Louisiana by the Geological Survey are assigned a number consisting of two parts, an abbreviation of the name of the parish in which the well is located and a consecutive well number. The number assigned does not indicate a specific location because the number is generally assigned in the order in which the well was inventoried. The prefix for Calcasieu Parish is CU; the prefix for Cameron Parish is $\mathrm{CN}$.

\section{HYDROLOGIC AND GEOLOGIC UNITS}

Descriptions of the hydrologic and geologic units have been thoroughly documented by Gabrysch and McAdoo (1972, p. 7-10) and Wesselman (1965, p. 1219). This report is primarily concerned with the Chicot aquifer which consists of alternating layers of sand and clay. The aquifer is divided into two units by $c l a y$ beds that in most instances separate an upper sand section from a lower sand section. Separation of these units is based on differences in permeability, water levels, and stratigraphic position. The lower unit of the Chicot aquifer is the principal source of ground water in Orange County and supplies about 94 percent of the ground water withdrawn. However, either unit could contain saltwater or be vulnerable to saltwater intrusion in the vicinity of the coast. 
The Evangeline aquifer underlies the Chicot aquifer but yields freshwater only in the central and northern part of the study area. This is typical of a coastal aquifer where the inland area of the aquifer contains freshwater and the coastal area contains saltwater. Regionally, the Evangeline aquifer is undeveloped in the report area, however, there is substantial local development at Evadale in Jasper County, Texas, and at Silsbee in Hardin County, Texas.

\section{GROUND-WATER DEVELOPMENT Pumpage}

Estimates of pumpage in the study area are based on questionnaires returned by major water users to the Texas Department of Water Resources. Estimated ground-water withdrawals from the lower unit of the Chicot aquifer during 196379 are given in table 1. The table shows that total ground-water pumpage in Orange County has changed liftle since 1963. Pumpage was $23.0 \mathrm{Mgal} / \mathrm{d}$ (million gallons per day) or $1.01 \mathrm{~m}^{3} / \mathrm{s}$ (cubic meters per second) for 1971 and 20.3 $\mathrm{Mgal} / \mathrm{d}(0.89 \mathrm{~m} / \mathrm{s})$ for 1979 , with an average (1971-79) of $21.2 \mathrm{Mgal} / \mathrm{d}$ (0.93 $\mathrm{m}^{3} / \mathrm{s}$ ). Pumpage from the upper unit of the Chicot aquifer (not included in table 1) averaged about $2 \mathrm{Mgal} / \mathrm{d}\left(0.088 \mathrm{~m}^{3} / \mathrm{s}\right)$ for $1971-79$. Al though the total yearly pumpage has changed little, the pumpage for municipal supply shows a generally steady increase while the pumpage for industrial use shows a generally steady decrease. The decrease in ground-water pumpage for industrial use primarily is due to the deterioration of ground-water quality from wells in the vicinity of freshwater-saltwater interfaces.

Al though the Evangeline aquifer is undeveloped in Orange and Jefferson Counties, it is developed at Evadale in Jasper County and at Silsbee in Hardin County. During 1979, about $25 \mathrm{Mgal} / \mathrm{d}\left(1.1 \mathrm{~m}^{3} / \mathrm{s}\right)$ of water was pumped from the aquifer at Evadale.

\section{Changes in Water Levels}

Fluctuations of water levels throughout the study area have varied because of changes in pumping patterns. Long-term fluctuations are shown in hydrographs of two wells in the area (fig. 3), well UJ-62-59-105 in eastern Orange County and well CU-530 in western Calcasieu Parish, Louisiana. Well UJ-62-59105 is located in an area that shows a water-level rise of about 1.6 feet $(0.49 \mathrm{~m})$ between 1971-80. Well CU-530 shows a water-level decline of 6.7 feet $(2.0 \mathrm{~m})$ for $1971-73$, and a subsequent rise to reflect a net water-level decline of 4.7 feet $(1.4 \mathrm{~m})$ for the report period.

Measurements of water levels upon which regional changes are based are made in the early part of the year when pumpage is minimal. Measurements made during 1975-80 are presented in table 2 (at back of report); measurements for 1971-74 were given by Bonnet (1975). The approximate altitudes of water levels (spring 1980) in wells screened in the lower unit of the Chicot aquifer are shown in figure 4 . Water levels for 1980 ranged from more than 40 feet $(12.2$ $\mathrm{m})$ above sea level in the southern parts of Jasper and Newton Counties to about 50 feet $(15.2 \mathrm{~m})$ below sea level in the vicinity of the city of Orange to about 90 feet $(27.4 \mathrm{~m})$ below sea level in south-central Orange County. The altitudes of water levels for 1971 were presented by Gabrysch and McAdoo (1972, fig. 5). 


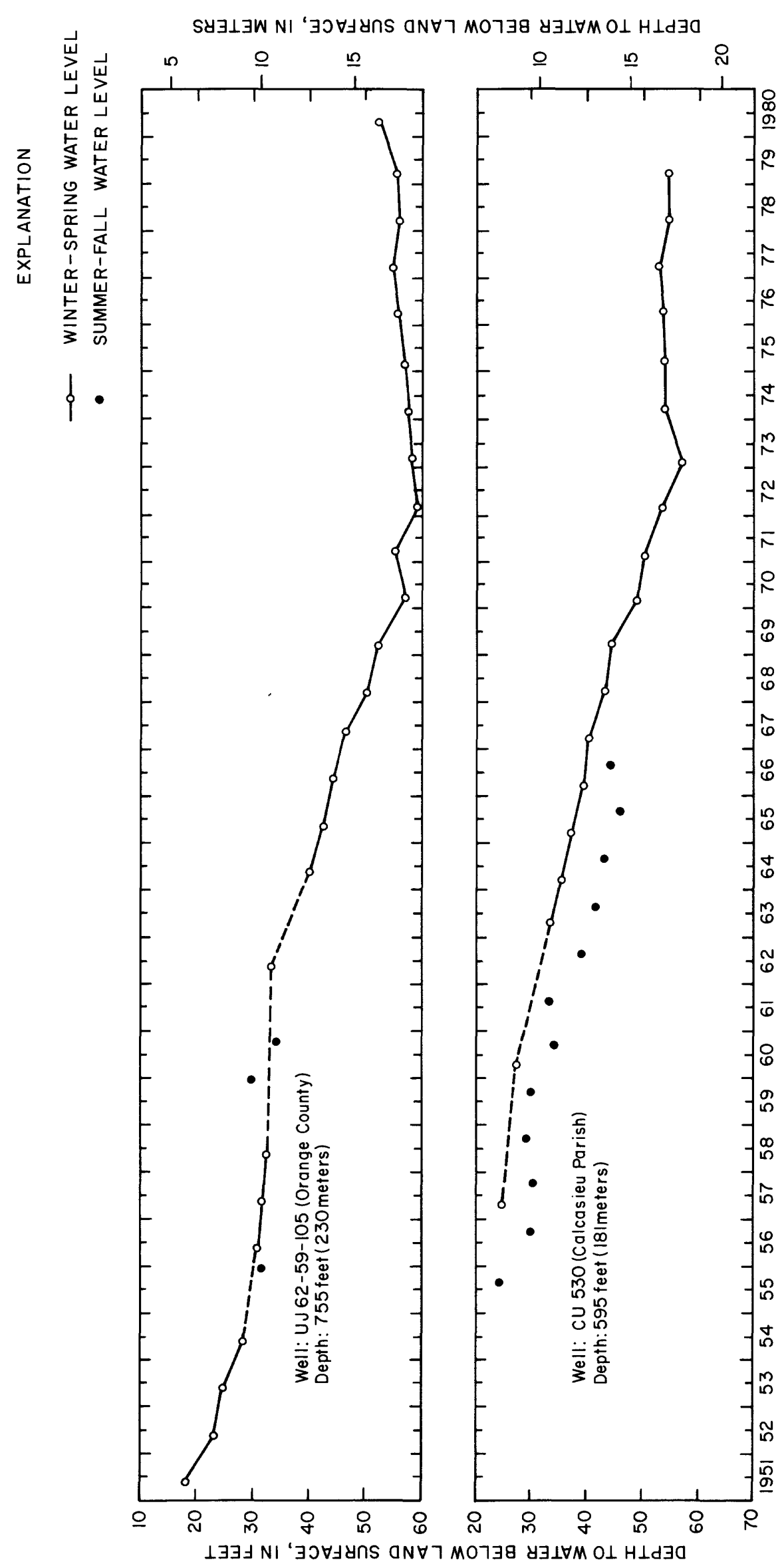

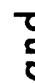

0
0
0
0
5
2
5
0
0
0
0
0
0
0

.

$\frac{2}{3}$

훙

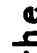

응

단

는

$\stackrel{0}{ \pm}$

.5

웅

는

$\frac{0}{8}$

$\stackrel{\circ}{=}$

.

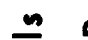

$\div$

은

$=$

$\frac{1}{2}$

!

$m$

ํำ 


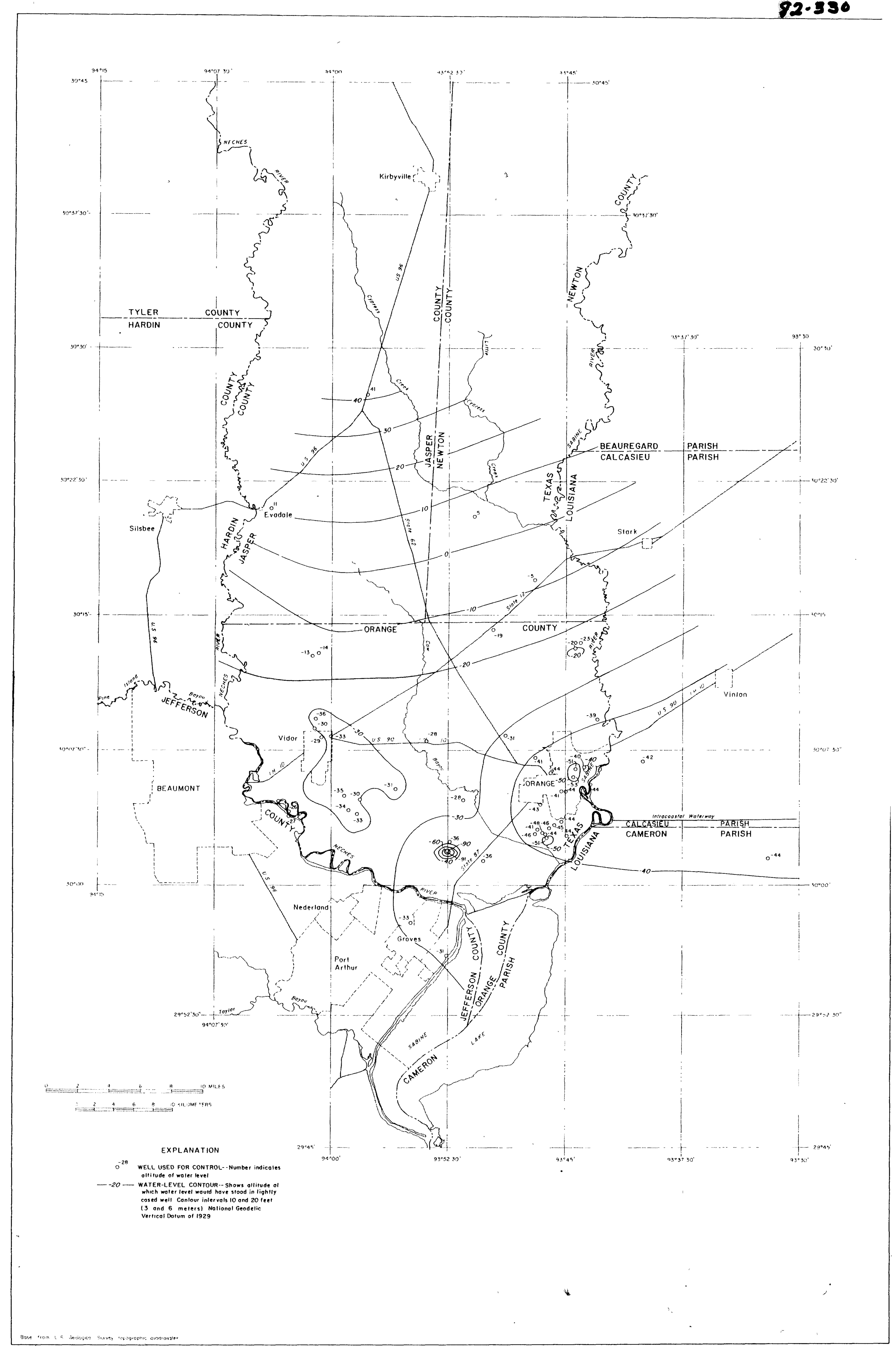


Table 1.--Pumpage of ground water from the lower unit of the

Chicot aquifer in Orange County, 1963-79

$$
\text { (million gallons per day) }
$$

\begin{tabular}{lccc}
\hline Year & $\begin{array}{c}\text { Municipa) } \\
\text { supply }\end{array}$ & $\begin{array}{c}\text { Industrial } \\
\text { use }\end{array}$ & Total \\
\hline 1963 & 3.8 & 14.7 & 18.5 \\
1964 & 4.1 & 16.2 & 20.3 \\
1965 & 4.5 & 16.3 & 20.8 \\
1966 & 4.7 & 16.3 & 21.0 \\
1967 & 5.8 & 14.7 & 20.5 \\
1968 & 4.6 & 16.6 & 21.2 \\
1969 & 4.8 & 16.0 & 20.8 \\
1970 & 5.1 & 15.9 & 21.0 \\
1971 & 5.4 & 17.6 & 23.0 \\
1972 & 5.4 & 17.7 & 23.1 \\
1973 & 5.3 & 15.8 & 21.1 \\
1974 & 5.5 & 15.0 & 20.5 \\
1975 & 5.7 & 12.8 & 18.5 \\
1976 & 5.9 & 15.7 & 21.6 \\
1977 & 6.1 & 16.2 & 22.3 \\
1978 & 6.4 & 13.6 & 20.0 \\
1979 & 7.0 & 13.3 & 20.3 \\
\hline
\end{tabular}


Net changes in the altitudes of water levels for this report period (fig. 5) are a result of differences determined from measurements made during spring 1971 and spring 1980. The map and measurements show the greatest water-level declines occurred in the south-central part of Orange County and southeast of Vidor. Based on these measurements, water levels declined 17.7 feet $(5.4 \mathrm{~m})$ in well UJ-62-57-904 and 11.1 feet $(3.4 \mathrm{~m})$ in well UJ-62-57-404. Water-level rises resulting from decreases in ground-water pumpage are evident in the industrial area southwest of the city of Orange. The largest rises in water levels were 14.0 feet $(4.3 \mathrm{~m})$ in well UJ-62-58-633 and 11.6 feet $(3.5 \mathrm{~m})$ in we11 UJ-62-58-613.

\section{Subsidence}

Regional subsidence in the study area has been small and is related primarily to the pumping of ground water with the consequent lowering of water levels and compaction of fine-grained sediments. The most widely used method to determine subsidence is comparison of bench-mark elevations for different time periods. Elevations of bench marks are not determined on a regular basis in the Orange County area. The latest elevations were determined during 1973 by the National Geodetic Survey. The location of bench marks and land-surface subsidence in the Orange County area for various time periods is shown in figure 6. Ratzlaff (1980) states that land-surface subsidence from 1953-55 to 1973 in Orange County generally was less than 0.5 foot $(0.15 \mathrm{~m})$. More than 0.5 foot $(0.15 \mathrm{~m})$ of subsidence occurred in the east-central area (city of Orange) and western area (city of Beaumont), where subsidence generally attributed to ground-water development ranged between 0.55 to 0.80 foot $(0.17$ to $0.24 \mathrm{~m})$. Most future large-scale water needs probably will be met by surfacewater supplies. If the use of surface water increases, additional regional subsidence is likely to be negligible.

Contrasting with the regional subsidence of the area is the local subsidence associated with the production of oil, gas, saltwater, and sulfur which exceeded 0.5 foot $(0.15 \mathrm{~m})$ in the Spindletop Dome and Port Acres gas field areas in Jefferson County, Texas. The estimated subsidence for the Spindletop Dome area during 1925-77 is shown in figure 7 and for the Port Acres gas field area during 1959-77 is shown in figure 8. Subsidence was determined by comparing elevations on 1977 topographic maps with those on 1925 maps. At Spindletop (fig. 7), subsidence is related to $0 i 1$ and gas development as well as a collapse feature due to sulfur mining. Ratzlaff estimated that of the 15 feet $(4.6 \mathrm{~m})$ of subsidence at Spindletop, 10 feet $(3.0 \mathrm{~m})$ may be due to sulfur mining. In the Port Acres gas field area (fig. 8), as much as 3 feet $(1.0 \mathrm{~m})$ of subsidence has occurred, probably as a result of oil, gas, and saitwater production.

\section{Water-Qual ity Changes}

The greatest threat to fresh ground-water resources in the study area is contamination by saltwater encroachment. Saltwater occurs along the coast and downdip of the freshwater. Saltwater encroachment in the lower unit of the Chicot aquifer has been observed in very local areas of the southeastern, southwestern, and south-central areas of the county. This encroachment occurs as inland and vertical migration of saltwater in response to ground-water withdrawals. A vertical or "coning upward" movement is most likely to occur when sands that contain freshwater and saltwater are not separated by clay beds. 


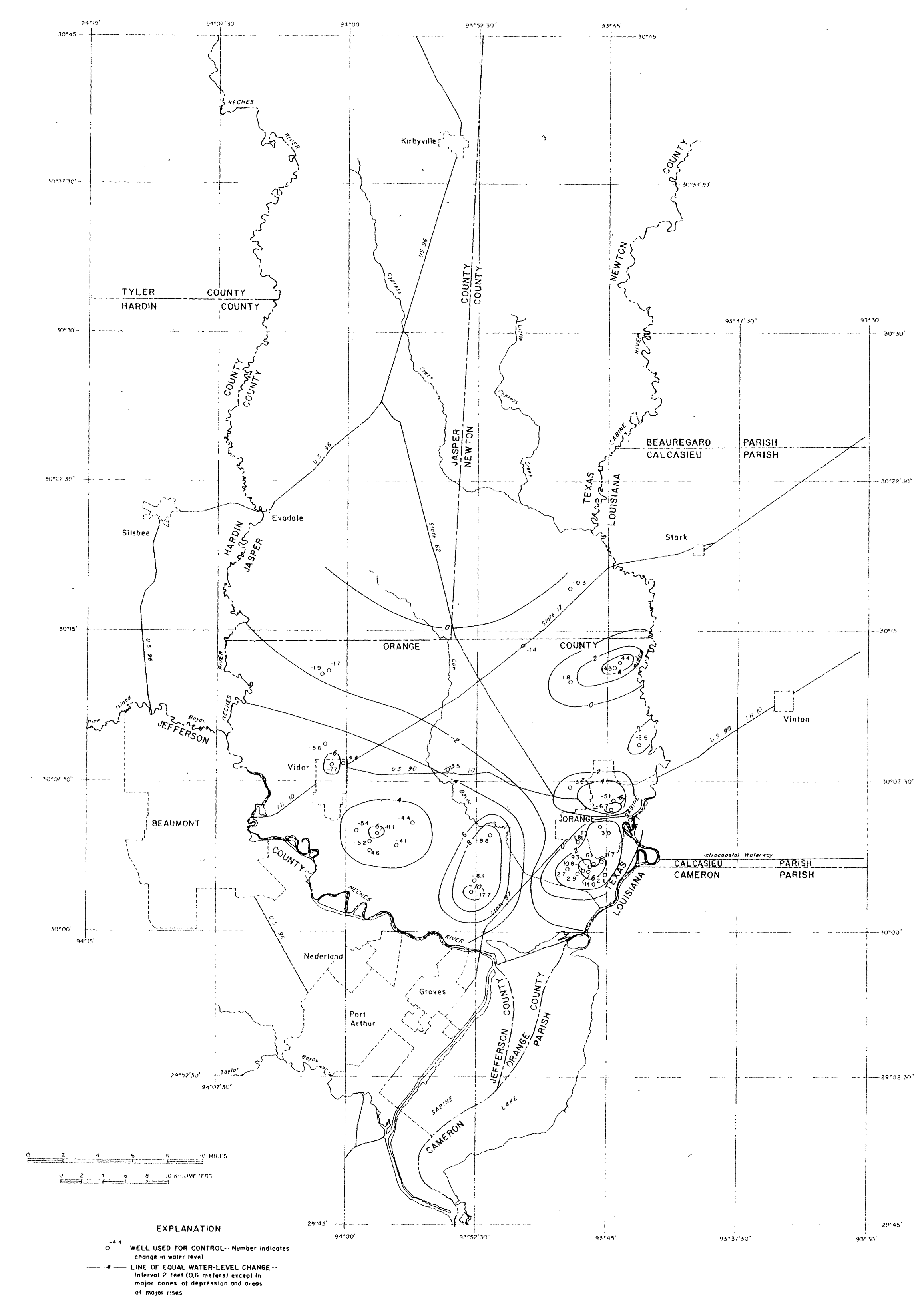



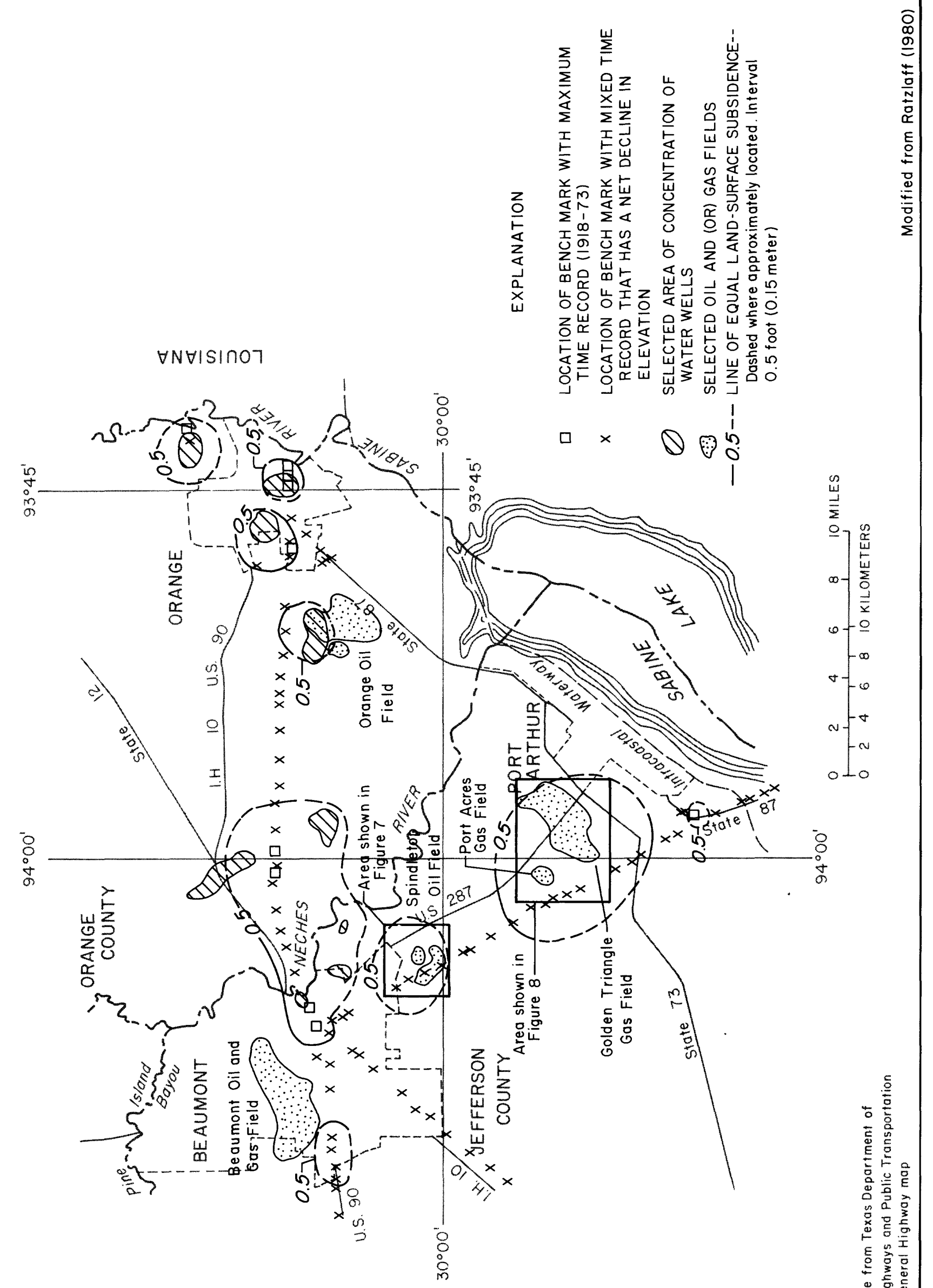

(2)

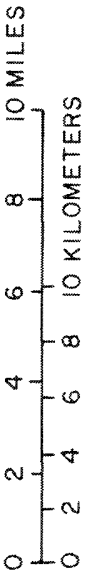

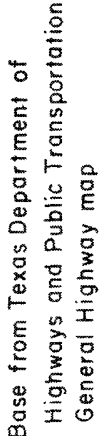




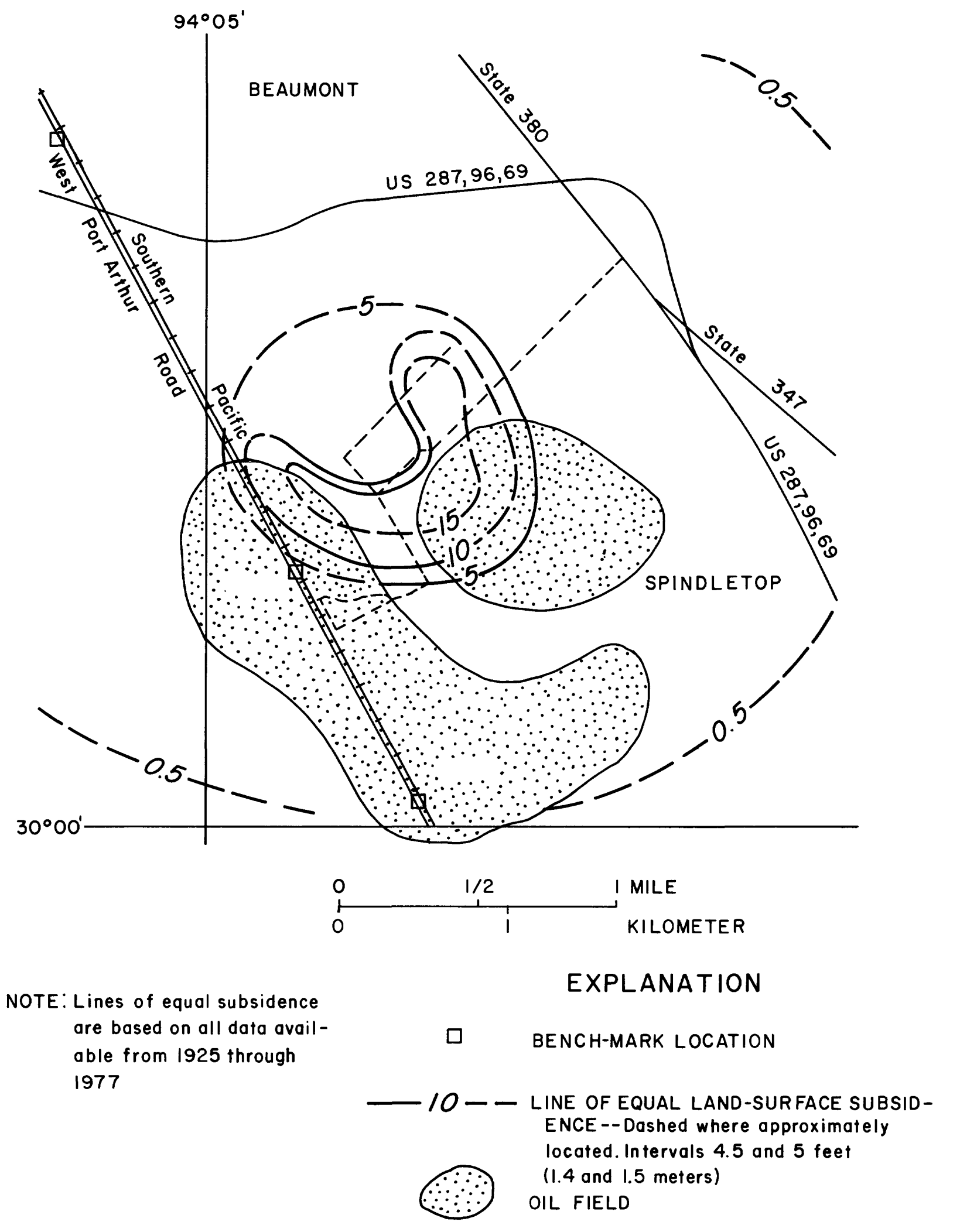

Base from U.S. Geological Survey topographic quadrangles

From Ratzlaff (1980)

Figure 7.-Land-surface subsidence in the Spindletop Dome area, Jefferson County, Texas, area, 1925-77 

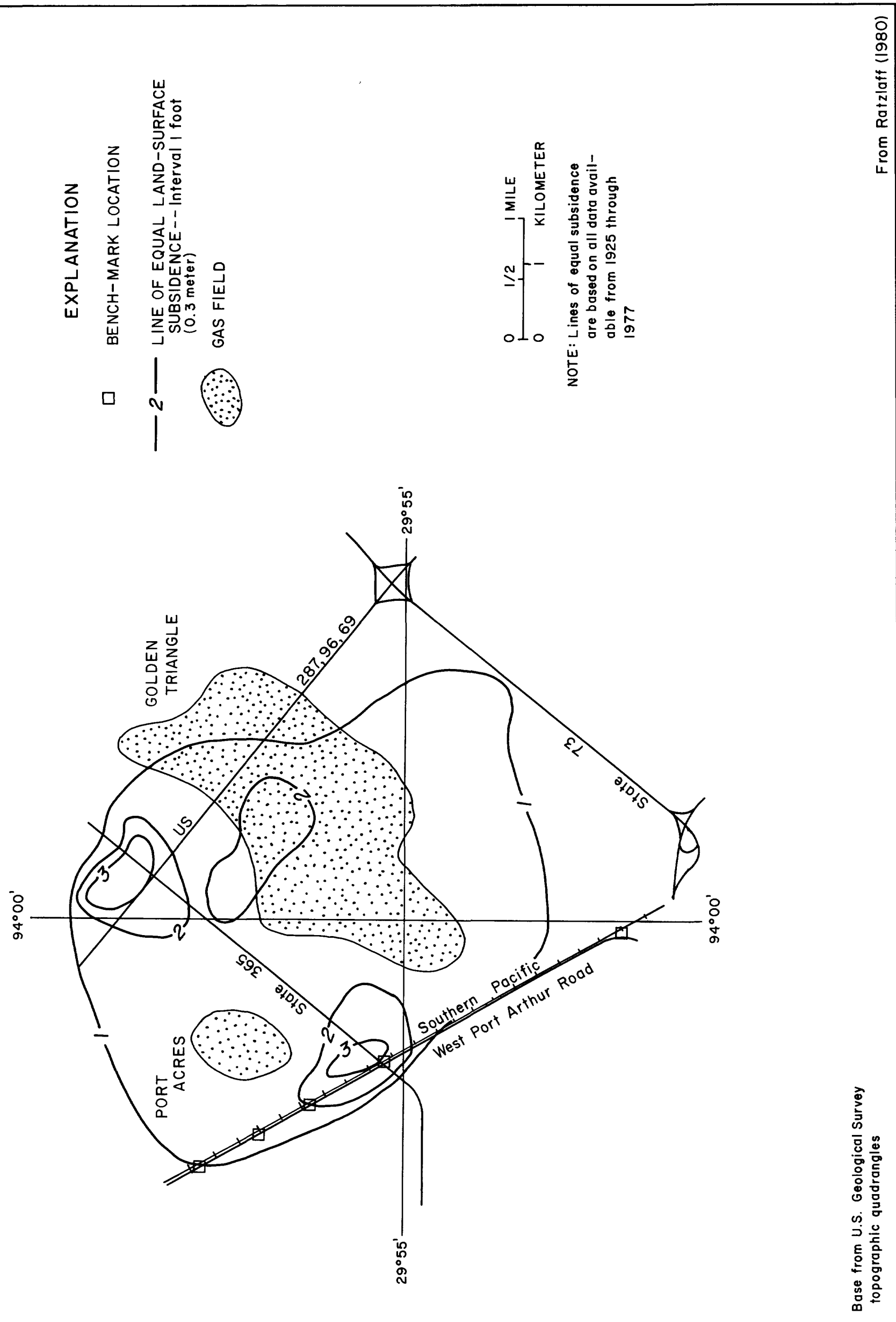

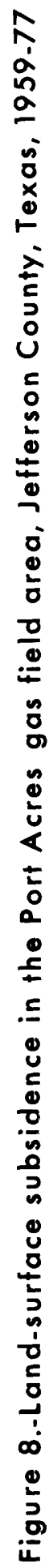


Two areas where saltwater encroachment has been documented by increases in chloride concentrations are in southwestern Orange County in the vicinity of Vidor (site A, fig. 9) and in southeastern Orange County south of the city of Orange (site B, fig. 11). Chemical analyses of water samples collected from 1975-79 are given in table 5 (at back of report). The location of the wells from which samples were collected are shown in figure 2.

Concentrations of chloride in water from six wells at site $A$ that were drilled during 1961 to supply water for industrial use in the southwestern part of the county are shown in figure 9. Analysis of water from one of the four deep wells, UJ-62-57-404, show that the chloride concentrations increased from 840 to $1,300 \mathrm{mg} / \mathrm{L}$ (milligrams per liter) between September 1972 and 0ctober 1979. The irregular chloride concentrations in water from the four deep wells indicate a "coning-up" of the saline water. Frequent sampling and analysis are used to monitor chloride concentrations of water from these wells. At the current (1980) level of development, the chloride concentrations are controlled by alternating well usage. Wells UJ-62-57-407 and UJ-62-57-408, which are drilled to a lesser depth, yield water with smaller chloride concentrations. The gradual increase in chloride concentrations in water from both wells between 1971-79 is shown in figure 10. Because of the regular pattern of slowly increasing chloride concentrations, the movement of saltwater is thought to be lateral toward the center of pumping as well as periodic "coning up" near the center of pumping. Pumpage from this southwest location averaged $3.2 \mathrm{Mgal} / \mathrm{d}\left(0.14 \mathrm{~m}^{3} / \mathrm{s}\right)$ between 1971-79.

The approximate concentrations of chloride in water from the lower unit of the Chicot aquifer at site B in 1972, 1974, 1976, and 1979 are shown in figure 11. Analysis of the data along the margin of the well field generally indicates a fluctuating of chloride concentrations. Water from well UJ-62-58-605, which is near the center of the well field, also had a fluctuating chloride concentration of $820 \mathrm{mg} / \mathrm{L}$ in September $1972,1,500 \mathrm{mg} / \mathrm{L}$ in October $1974,1,400$ $\mathrm{mg} / \mathrm{L}$ in 0ctober 1976, and $1,300 \mathrm{mg} / \mathrm{L}$ in 0ctober-November 1979. This fluctuating trend in chloride concentrations is probably due to "coning up" of deeper saltwater and is in response to changing rates of ground-water pumping in the area. This is the same pattern that was observed at site A.

In south-central Orange County there is a network of seven industrial wells that sometimes yield water containing excessive concentrations of chloride. Water from well UJ-62-57-901 (fig. 2), that is used only for emergencies, had a chloride concentration of $1,180 \mathrm{mg} / \mathrm{L}$ on December 27,1979 (table 5). The irregular pattern of chloride concentrations in water from the seven wells indicates vertical movement of saline water. Pumpage from this southcentral industrial location averaged $1.3 \mathrm{Mgal} / \mathrm{d}\left(0.057 \mathrm{~m}^{3} / \mathrm{s}\right)$ from 1971-79.

\section{Ground-Water Problems and Future Development}

Saltwater encroachment is the principal ground-water problem in the study area. Fortunately, it is limited to the coastal area and generally to deeper wells. In this area, the chloride concentations can be expected to increase due to lateral encroachment and vertical "coning up" if a well field is pumped continually and has a concentrated center of pumping. However, if the withdrawals are moderate or decreased, as needed to control the chloride concentrations, substantial quantities of fresh ground water can be pumped near the 

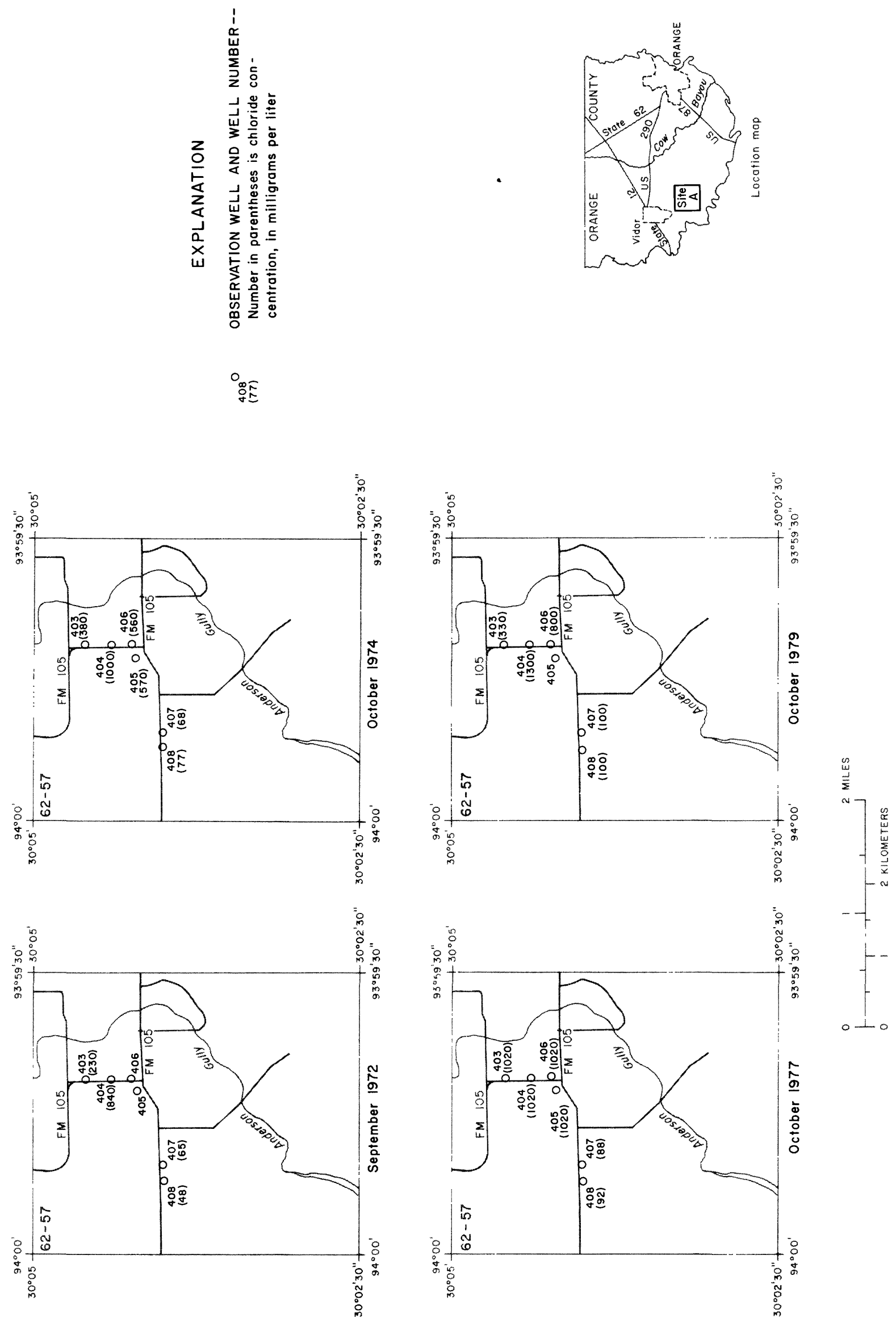


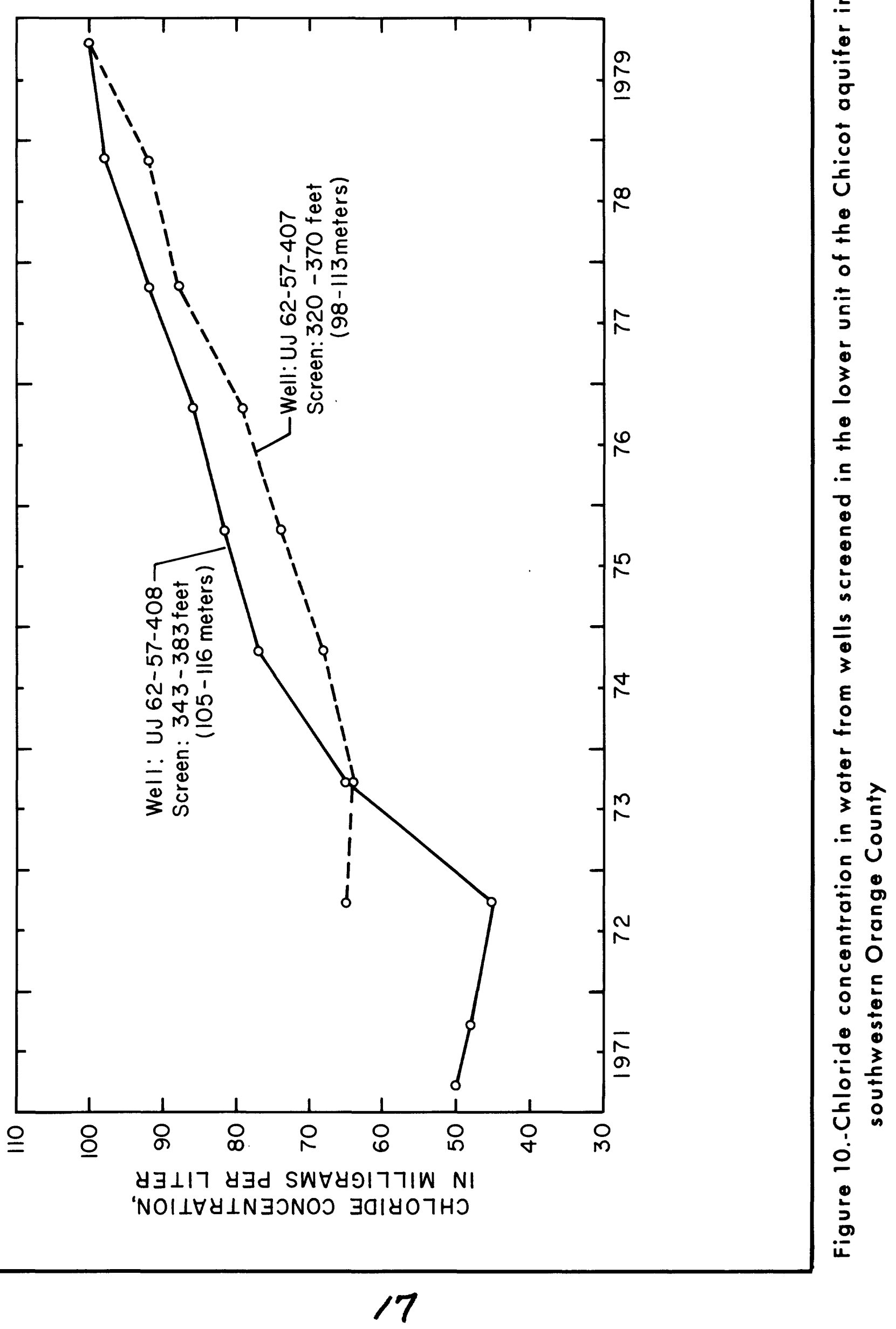



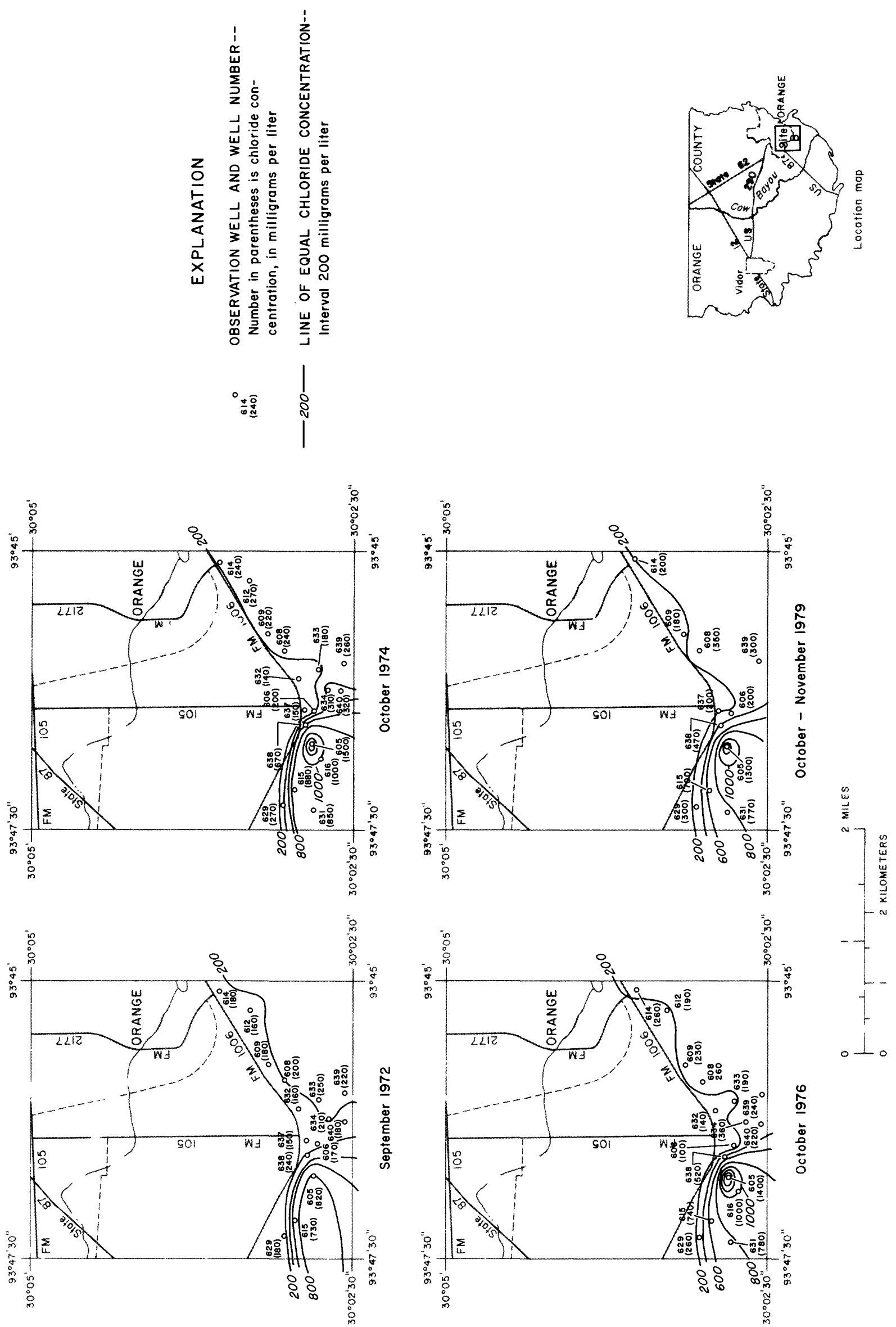
coast. Monitoring the movement of saltwater in the aquifer, adjusting the pumping rates and pattern, and distributing the pumping throughout large areas are of primary importance in keeping the chloride concentrations less than unacceptably large values.

The ground-water resources of Orange County are only partly developed. Wesselman (1965) states that abundant, accessible water underlying Orange County is equal to about four times the present (1980) daily pumpage. To properly manage future ground-water development, the continued collection of hydrologic data is needed. These data will provide information for predicting future water levels and developing plans to minimize saltwater encroachment and landsurface subsidence.

\section{SUMMARY}

Water-quality data indicate that the chloride concentration of the ground water is increasing in the southeastern, southwestern, and south-central areas of Orange County. The chloride concentration increased as the saltwater interface moved toward the areas of heaviest withdrawal.

Ground-water withdrawals of $20 \mathrm{Mgal} / \mathrm{d}\left(0.88 \mathrm{~m}^{3} / \mathrm{s}\right)$ during 1980 were about equal to that reported in 1965. Municipal ground-water use has increased from $4.5 \mathrm{Mgal} / \mathrm{d}\left(0.20 \mathrm{~m}^{3} / \mathrm{s}\right)$ during 1965 to $7.0 \mathrm{Mgal} / \mathrm{d}\left(0.31 \mathrm{~m}^{3} / \mathrm{s}\right)$ during 1979 , while industrial use has decreased from $16.3 \mathrm{Mgal} / \mathrm{d}(0.71 \mathrm{~m} / \mathrm{s})$ during 1965 to 13.3 $\mathrm{Mgal} / \mathrm{d}\left(0.58 \mathrm{~m}^{3} / \mathrm{s}\right)$ during 1979.

Comparison of elevations of bench marks determined during 1973 and 1918 show regional land-surface subsidence was less than 0.5 foot $(0.15 \mathrm{~m})$. Because water-level declines have been small, there has been little, if any, additional land-surface subsidence since 1973.

Water levels declined in most of the study area during 1971-80, generally at a slower rate than before 1971. Water levels tended to stabilize in areas where ground-water withdrawals decreased, and in eastern Orange County, water levels rose as much as 14 feet $(4.3 \mathrm{~m})$. 
Baker, E. T., Jr., 1964, Geology and ground-water resources of Hardin County, Texas: Texas Water Commission Bul let in 6406, $179 \mathrm{p}$.

Bonnet, C. W., 1975, Ground-water data for Orange County and vicinity, Texas and Louisiana, 1971-74: Texas Water Development. Board Report 197, 26 p. Gabrysch, R. K., and McAdoo, G. D., 1972, Development of ground-water resources in the Orange County area, Texas and Louisiana, 1963-71: Texas Water Development Board Report 156, $47 \mathrm{p}$.

Harder, A. H, 1960, The geology and ground-water resources of Calcasieu Parish, Louisiana: U.S. Geological Survey Water-Supply Paper 1488, 102 p.

Jones, P. H., Hendricks, E. L., Irelan, Burdge, and others, 1956, Water resources of southwestern Louisiana: U.S. Geological Survey Water-Supply Paper $1364,460 \mathrm{p}$.

Jones, P. H., Turcan, A. N., Jr., and Skibitzke, H. E., 1954, Geology and ground-water resources of southwestern Louisiana: Louisiana Department of Conservation, Geological Survey Bulletin 30, 285 p.

McAdoo, G. E., 1968, Ground-water data for Orange County and vicinity, Texas and Louisiana, 1968: U.S. Geological Survey open-file report, 20 p.

1969, Ground-water data for Orange County and vicinity, Texas and Louisiana, 1969: U.S. Geological Survey open-file report, $22 \mathrm{p}$.

1970, Ground-water data for Orange County and vicinity, Texas and Louisiana, 1970: U.S. Geological Survey open-file report, $19 \mathrm{p}$.

Ratzlaff, K. W., 1980, Land-surface subsidence in the Texas coastal region: U.S. Geological Survey Open-File Report 80-969, 19 p.

Turcan, A. N., Jr., Wesselman, J. B., and Chabot, Kilburn, 1966, Interstate correlation of aquifers, southwestern Louisiana and southeastern Texas: U.S. Geological Survey Professional Paper 550-D, p. D231-236.

Wesselman, J. B., 1965, Geology and ground-water resources of Orange County, Texas: Texas Water Commission Bullet in 6515, $112 \mathrm{p}$. 1967, Ground-water resources of Jasper and Newton Counties, Texas: Texas Water Development Board Report 59, $177 \mathrm{p}$. 1971, Ground-water resources of Chambers and Jefferson Counties, Texas, with a section on Quaternary geology, by Saul Aronow: Texas Water Deve 1opment Board Report 133, $183 \mathrm{p}$.

Winslow, A. G., and Kister, L. R., 1956, Saline-water resources of Texas: U.S. Geological Survey Water-Supply Paper 1365, $105 \mathrm{p}$.

Winslow, A. G., and Wood, L. A., 1959, Relation of land subsidence to groundwater withdrawals in the upper Gulf Coast region, Texas: Mining Engineering, vol. 11, no. 10, p. 1030-1034.

Wood, L. A., and Gabrysch, R. K., 1965, Analog model study of ground water in the Houston district, Texas, with a section on Design, construction, and use of electric analog models, by E. P. Patten, Jr.: Texas Water Commission Bul letin 6508, $103 \mathrm{p}$.

Zack, A. L., 1971, Ground-water pumpage and related effects, southwestern Louisiana, 1970, with a section on Surface-water withdrawals: Louisiana Water Resources pamphlet no. 27,35 p. 
SUPPLEMENTAL INFORMATION

$-21-$ 
Table 2.--Water levels in observation wells in the study area, 1975-80

(feet below land surface)

\section{ORANGE COUNTY, TEXAS}

$\begin{array}{ll}\text { Water } & \text { Wevel }\end{array}$

WELL UJ-61-56-103

Owner: B. H. Thibodeau

Depth: 76 feet

Elevation: 23 feet

Mar. 10, 1975

12.40

Mar. 17, 1976

11.09

Mar. 28, 1977

11.83

Apr. 3, 1978

12.54

Mar. 27, 1979

10.66

Mar. 31, 1980

7.31

WELL UJ-61-56-116

Owner: H. H. Houseman

Depth: 800 feet

Elevation: 21 feet

Mar. 10, 1975

38.11

Mar. 24, 1976

38.38

Mar. 28, 1977

38.93

Apr. 3,1978

40.56

Mar. 27, 1979

41.73

Mar. 31,1980

41.28

WELL UJ-61-56-314

Owner: L. B. Williamson

Screen: $375-385$ feet

Elevation: 27 feet

Mar. 10, 1975

39.48

Mar. 26, 1976

38.95

Mar. 28, 1977

39.90

Apr. 3, 1978

41.48

Mar. 27, 1979

42.29

Mar. 31,1980

41.33

WELL UJ-64-56-315

Owner: Iwanda Trailer Park

Screen: $356-380$ feet

Elevation: 29 feet

Mar. 10, 1975

39.50

Mar. 24, 1976

38.69
Date

Water

WELL UJ-61-56-315--Cont.

Mar. 28, 1977

39.96

Apr. 3, 1978

41.31

Mar. 31, 1980

41.63

WELL UJ-61-56-611

Owner: B\&B Water System

Screen: 441-457 feet

Elevation: 22 feet

Mar. 24, 1976

43.96

Mar. 28, 1977

46.18

Apr. 3, 1978

48.40

Mar. 27, 1979

51.20

WELL UJ-61-56-901

Owner: Orange County, WC\&ID No. 1, well 2

Screen: $350-400$ feet

Elevation: 21 feet

Mar. 12, 1975

42.02

Mar. 23, 1976

46.13

Apr. 1, 1977

49.04

Apr. 7, 1978

47.15

Mar. 30, 1979

44.81

Apr. 8,1980

50.02

WELL UJ-61-56-911

Owner: B\&B Water Systems

Screen: $468-486$ feet

Elevation: 12 feet

Mar. 10, 1975

39.04

Mar. 23, 1976

39.30

Mar. 22, 1977

40.10

Apr. 3, 1978

40.30

Mar. 27, 1979

41.65
Date

Water

WELL UJ-61-56-919

Owner: Orange County WC\&ID No. 1, well 13

Screen: $385-420$ feet

Elevation: 21 feet

Mar. 12, 1975

Mar. 23, 1976

Apr. 1, 1977

50.42

Apr. 7, 1978

51.57

Mar. 30, 1979

51.67

Apr. 8, 1980

54.34

WELL UJ-61-56-920

Owner: B\&B Water System

Depth: 380 feet

Elevation: 11 feet

Mar. 10, 1975

44.82

Mar. 23, 1976

45.40

Mar. 28, 1977

45.35

Mar. 27, 1978

45.49

Mar. 27, 1979

48.13

Apr. 1,1980

46.53

WELL UJ-62-49-503

Owner: G. L. Linscomb

Depth: 117 feet

Elevation: 26 feet

Mar. 12, 1975

8.98

Mar. 25, 1976

8.44

Mar. 23, 1977

8.58

Mar. 31, 1978

9.40

Mar. 27, 1979

9.08

Apr. 1, 1980

WELL UJ-62-49-904

Owner: Texas Dept. of Highways \& Public Transportation Screen: $399-415$ feet

Elevation: 16 feet

Mar. 10, 1975

42.61

Mar. 23, 1976

43.00 
Table 2.--Water levels in observation wells in the study area, 1975-80--Continued

$\begin{array}{ll} & \text { Water } \\ \text { Date } & \text { level }\end{array}$

WELL UJ-62-49-904--Cont.

\begin{tabular}{ll} 
Mar. 21, 1977 & 43.24 \\
Mar. 27, 1978 & 43.36 \\
Mar. 26, 1979 & 44.48 \\
Apr. 3,1980 & 43.90 \\
\multicolumn{3}{c}{ WELL UJ-62-50-201 } \\
Owner: Boyce Ward \\
Screen: $476-586$ feet \\
Elevation: 26 feet \\
Mar. 12, 1975 \\
Mar. 4, 1976 & 44.60 \\
Mar. 23, 1977 & 44.61 \\
Apr. 4, 1978 & 44.42 \\
Mar. 27, 1979 & 45.25 \\
Apr. 1, 1980 & 46.00
\end{tabular}

\begin{tabular}{ll}
\multicolumn{3}{c}{ WELL UJ-62-50-807 } \\
Owner: Frank Michell \\
Screen: $442-454$ feet \\
Elevation: 20 feet \\
Mar. 11, 1975 & 49.01 \\
Mar. 23, 1976 & 49.29 \\
Mar. 25, 1977 & 49.19 \\
Mar. 30, 1978 & 49.41 \\
Mar. 28, 1979 & 50.41 \\
Apr. 3, 1980 & 50.02
\end{tabular}

WELL UJ-62-50-808

Owner: H. D. Womack

Depth: 643-655 feet

Elevation: 21 feet

$\begin{array}{ll}\text { Mar. 11, } 1975 & 51.90 \\ \text { Mar. 23, } 1976 & 52.45 \\ \text { Mar. 25, 1977 } & 52.06 \\ \text { Mar. 30, 1978 } & 52.3 \\ \text { Mar. 28, 1979 } & 53.28 \\ \text { Apr. 3, } 1980 & 51.90\end{array}$

Water

WELL UJ-62-50-904

Owner: George Gl idden

Depth: 566 feet

Elevation: 12 feet

Mar. 12, 1975

6.83

Mar. 26, 1976

6.45

Mar. 23, 1977

6.28

Mar. 28, 1978

6.41

Mar. 30, 1979

6.83

Apr. 1, 1980

5.65

WELL UJ-62-51-103

Owner: Owens I1linois, Inc. Screen: 445-515 feet Elevation: 25 feet

Mar. 12, 1975

46.38

Mar. 25, 1976

45.51

Mar. 23, 1977

45.55

Mar. 28, 1978

46.41

Apr. 3, 1980

45.05

WELL UJ-62-51-104

Owner: Owens Illinois, Inc. Screen: 460-470 feet Elevation: 25 feet

Mar. 12, 1975

48.66

Mar. 25, 1976

48.20

Mar. 23, 1977

47.02

Mar. 28, 1978

49.03

Mar. 30, 1979

49.39

Apr. 3,1980

47.60

WELL UJ-62-51-707

Owner: Phillips Chemical Co. Screen: $428-488$ feet Elevation: 12 feet

Mar. 12, 1975

52.56

Mar. 25, 1976

49.93

Mar. 23, 1977

49.65

Mar. 28, 1978

50.76
Date

Water level

WELL UJ-62-51-707--Cont.

Mar. 30, 1979

51.97

Apr. 3,1980

50.96

WELL UJ-62-57-203

Owner: K. Kishi

Depth: 740 feet

Elevation: 18 feet

Mar. 18, 1975

46.89

Mar. 24, 1976

47.29

Mar. 22, 1977

48.00

Mar. 28, 1978

47.89

Mar. 28, 1979

49.38

Apr. 9,1980

48.90

WELL UJ-62-57-401

Owner: Texas Eastern Trans. Co.

Screen: 448-468 feet

Elevation: 16 feet

Mar. 18, 1975

46.58

Mar. 24, 1976

52.30

Mar. 22, 1977

47.82

Mar. 28, 1978

49.09

Mar. 28, 1979

49.57

Apr. 2, 1980

48.91

WELL UJ-62-57-403

Owner: Gulf States Util. Co. Screen: 433-483 feet

Elevation: 15 feet

Mar. 21, 1975

43.73

Mar. 25, 1976

45.66

Mar. 30, 1977

46.16

Apr. 25, 1977

45.2

Mar. 28, 1978

41.43

Mar. 28, 1979

48.02

Apr. 11, 1980

45.30 
Table 2.--Water levels in observation wells in the study area, 1975-80--Continued

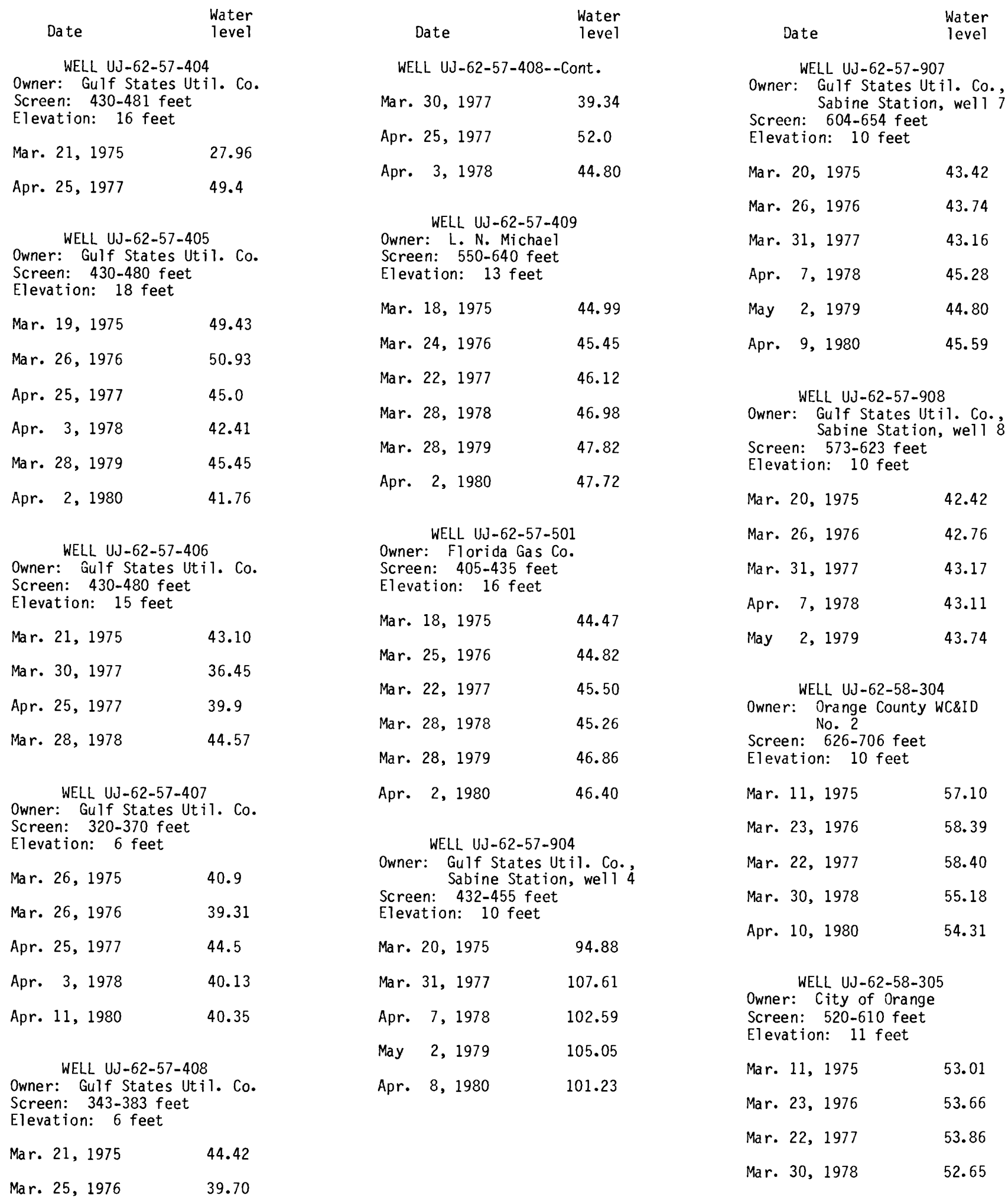


Table 2.--Water levels in observation wells in the study area, 1975-80--Continued

$$
\begin{array}{ll}
\text { Water } & \\
\text { Dater } & \text { level }
\end{array}
$$

WELL UJ-62-58-305--Cont.

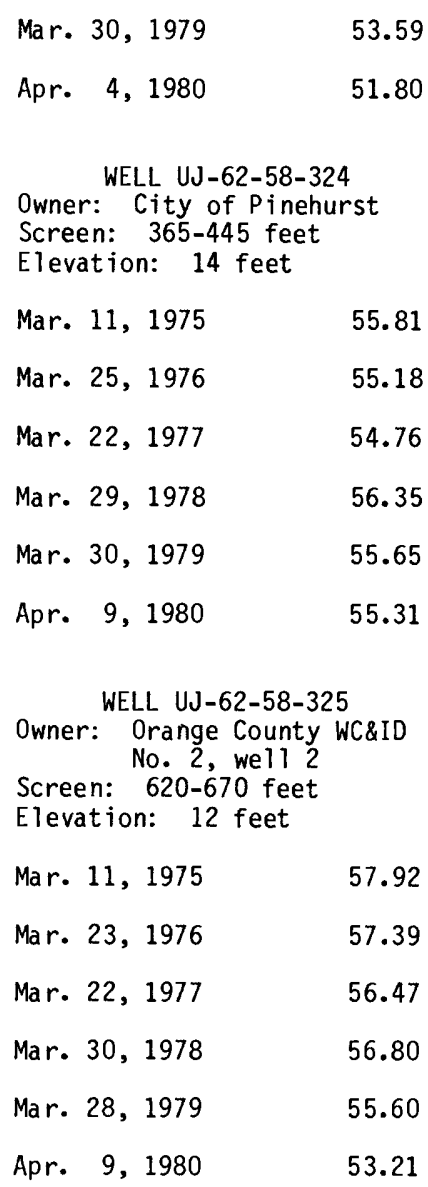

WELL UJ-62-58-403

Owner: Orangefield Ind. School Dist.

Screen: $460-480$ feet

Elevation: 15 feet

$\begin{array}{ll}\text { Mar. 18, 1975 } & 39.85 \\ \text { Mar. 24, 1976 } & 41.02 \\ \text { Mar. 22, 1977 } & 41.40 \\ \text { Mar. 28, 1978 } & 41.57 \\ \text { Mar. 28, 1979 } & 41.61 \\ \text { Apr. 3, 1980 } & 42.45\end{array}$

$\begin{array}{ll}\text { Water } & \text { Wate } \\ \text { level }\end{array}$

WELL UJ-62-58-410

Owner: Orangefield Rec. Park

Screen: $110-120$ feet

Elevation: 5 feet

Mar. 18, 1975

4.25

Mar. 24, 1976

4.24

Mar. 22, 1977

7.20

Mar. 28, 1978

4.25

Mar. 28, 1979

6.28

Apr. 3, 1980

6.89

WELL UJ-62-58-602

Owner: Donnar Corp.

Depth: 711 feet

Elevation: 14 feet

Mar. 18, 1975

58.65

Mar. 23, 1976

60.87

Mar. 30, 1977

56.28

Mar. 28, 1978

57.85

Mar. 28, 1979

59.13

Apr. 3, 1980

57.12

WELL UJ-62-58-603

Owner: W. H. Stark Est.

Depth: 204 feet

Elevation: 8 feet

Mar. 18, 1975

Mar. 22, 1976

8.60

Mar. 21, 1977

8.51

Mar. 27, 1978

8.76

Mar. 27, 1979

8.82

Apr. 4, 1980

8.28

WELL UJ-62-58-605

Owner: Gulf Chemical Co., well 4

Screen: $604-717$ feet

Elevation: 7 feet

Mar. 21, 1975

58.09

Mar. 23, 1976

63.13

Mar. 24, 1977

61.30

Mar. 31, 1978

59.91
Date

Water

WELL UJ-62-58-605--Cont.

Mar. 28, 1979

60.46

Apr. 10,1980

55.04

WELL UJ-62-58-608

Owner: Allied Chemical Co.

Screen: $620-735$ feet

Elevation: 8 feet

Mar. 29, 1978

55.02

Mar. 27, 1979

55.90

Apr. 4, 1980

54.43

WELL UJ-62-58-609

Owner: E. I. DuPont Co. well 103-3

Screen: 634-723 feet

Elevation: 5 feet

Apr. 6, 1978

55.26

Mar. 28, 1979

55.87

Apr. 11, 1980

51.90

WELL UJ-62-58-610

Owner: E. I. DuPont Co., well 103-3.1

Depth: 715 feet

Elevation: 7 feet

Mar. 21, 1975

60.05

Mar. 24, 1976

64.22

Apr. 1, 1977

59.95

Apr. 6, 1978

55.19

Mar. 28, 1979

55.56

Apr. 11, 1980

51.85

WELI UJ-62-58-611

Owner: E. I. DuPont Co., well 103-2

Depth: 715 feet Elevation: 8 feet

Mar. 18, 1975

59.16

Mar. 23, 1976

62.83

Mar. 21, 1977

60.81

Mar. 27, 1978

55.90

Mar. 26, 1979

55.36

Mar. 26, 1980

53.4 
Table 2.--Water levels in observation wells in the study area, 1975-80--Continued

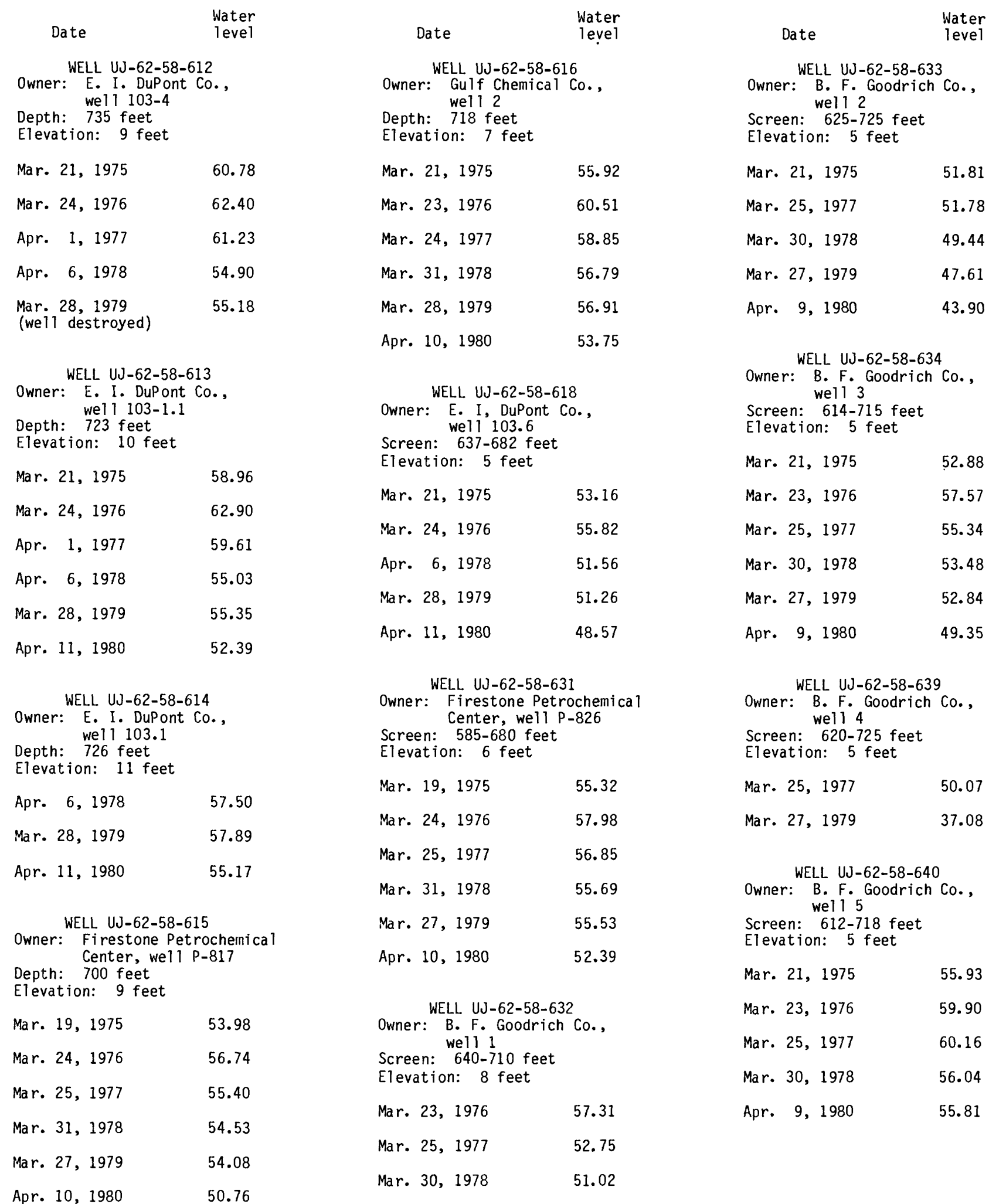


Table 2.--Water levels in observation wells in the study area, 1975-80--Continued

$$
\begin{array}{ll}
\text { Water } \\
\text { Date }
\end{array}
$$

WELL UJ-62-58-641

Owner: E. I. DuPont Co., well 103-6

Screen: 697-702 feet

Elevation: 5 feet

Mar. 21, 1975

53.68

Mar. 24, 1976

56.36

Apr. 1,1977

54.00

Apr. 6, 1978

51.89

Mar. 28, 1979

52.1

Apr. 11, 1980

49.08

\section{WELL UJ-62-58-702 \\ Owner: Orange County WC\&ID No. 3, well 2 \\ Screen: 600-672 feet}

Elevation: 10 feet

Mar. 19, 1975

48.38

Mar. 26, 1976

48.17

Mar. 30, 1977

47.48

Mar. 29, 1978

46.30

Mar. 27, 1979

47.76

Apr. 3, 1980

45.67

WELL UJ-62-58-809

Owner: Orange County WC\&ID No. 3 , well 3

Screen: $570-650$ feet

Elevation: 7 feet

Mar. 19, 1975

Mar. 26, 1976

Mar. 3, 1977

47

Mar. 29, 1978

Mar. 27, 1979

50.54

WELL UJ-62-58-810 Owner: H. H. Silkwood Screen: $160-170$ feet

Elevation: 5 feet

Mar. 19, 1975

Mar. 23, 1976

9.28

Mar. 30, 1977

9.75

Mar. 29, 1978

9.80
Date Water

level

WELL UJ-62-58-810--Cont.

$\begin{array}{ll}\text { Mar. 27, } 1979 & 9.74 \\ \text { Apr. 9, } 1980 & 9.02\end{array}$

WELL UJ-62-59-101

Owner: City of Orange, well 7

Screen: 555-666 feet

Elevation: 10 feet

Mar. 11, 1975

58.66

Mar. 23, 1976

62.52

Mar. 22, 1977

59.65

Mar. 30, 1978

61.61

Mar. 30, 1979

65.70

Apr. 10, 1980

61.36

WELL UJ-62-59-103

Owner: City of Orange, well 2

Screen: 565-685 feet

Elevation: 9 feet

Mar. 11, 1975

60.19

Mar. 23, 1976

60.42

Mar. 14, 1977

57.1

Mar. 30, 1978

58.29

Mar. 30, 1979

61.93

Apr. 4,1980

62.63

WELL UJ-62-59-105

Owner: Levingston Shipyard

Screen: $672-737$ feet

Elevation: 9 feet

Mar. 11, 1975

56.94

Mar. 22, 1976

56.93

Mar. 21, 1977

55.70

Mar. 27, 1978

54.40

Mar. 26, 1979

56.39

Apr. 1, 1980

53.24
Date

Water

WELL UJ-62-59-123

Owner: City of Orange, well 9

Screen: 529-643 feet

Elevation: 10 feet

Mar. 11, 1975

53.93

Mar. 23, 1976

55.86

Mar. 22, 1977

55.78

Mar. 30, 1978

53.55

Mar. 30, 1979

53.89

Apr. 10, 1980

50.45 
Table 2.--Water levels in observation wells in the study area, 1975-80--Continued

HARDIN COUNTY, TEXAS

Water Date

level

WELL LH-61-47-208

Owner: City of Silsbee

Screen: 442-842 feet

Elevation: 80 feet

$\begin{array}{lr}\text { Mar. 25, 1975 } & 93.14 \\ \text { Mar. 18, 1976 } & 99.30 \\ \text { Mar. 29, 1977 } & 97.13 \\ \text { Apr. 4, 1978 } & 100.40 \\ \text { Mar. 29, 1979 } & 101.42 \\ \text { Apr. 7, 1980 } & 97.90\end{array}$

WELL LH-61-47-210

Owner: City of Silsbee

Depth: 900 feet

Elevation: 87 feet

Mar. 25, 1975

107.10

Feb. 18,1976

117.22

Mar. 29, 1977

104.86

Apr. 4, 1978

112.52

Mar. 29, 1979

115.65

Apr. 7, 1980

114.03

WELL. LH-61-47-304

Owner: City of Silsbee, well 4

Screen: 595-905 feet

Elevation: 80 feet

Mar. 29, 1979

Apr. 7,1980

90.61

WELL LH-61-47-804

Owner: Lumberton Water Supply Corp.

Screen: 395-458 feet

El evation: 55 feet

Apr. 4, 1978

45.02

Mar. 29, 1979

44.44

Apr. 7, 1980

58.10

\section{JASPER COUNTY, TEXAS}

Water

Date

Date

Water

WELL LH-61-55-203

Owner: City of Beaumont

Screen: 301-775 feet

Elevation: 27 feet

Mar. 25, 1975

64.70

Mar. 17, 1976

55.58

Apr. 5, 1978

58.79

Mar. 29, 1979

61.45

Apr. 7, 1980

58.32

WELL LH-61-55-204

Owner: City of Beaumont

Screen: 311-780 feet

Elevation: 25 feet

Mar. 25, 1975

48.93

Mar. 26, 1976

49.82

Mar. 29, 1977

56.42

WELL LH-61-55-206

Owner: Lumberton Water Supply Corp., well 1

Screen: $380-443$ feet

Elevation: 35 feet

55.24

Apr. 4,1978

65.00

Mar. 29, 1979

59.21

Apr. 7, 1980

WELL PR-6I-48-209

Owner: East Texas Pulp and

Paper Co.

Screen: 213-594 feet

Elevation: 45 feet

Dec. 6, 1975

35.40

Dec. 3,1976

36.00

Dec. 3, 1977

39.60

Dec. 6, 1978

39.14

June 17,1980

33.30

WELL PR-61-48-214

Owner: Southern Pine Co.

Depth: 226 feet

Elevation: 42 feet

Mar. 25, 1975

32.58

Mar. 17, 1976

35.48

Mar. 28, 1977

34.40

Apr. 4, 1978

36.25

Mar. 29, 1979

35.10

Apr. 8, 1980

35.60

WELI PR-61-48-221

Owner: East Texas Pulp and Paper Co.

Screen: $723-1,264$ feet

Elevation: 45 feet

Dec. 6, 1975

189.61

Dec. 8,1976

185.90

Dec. 8,1977

187.45

Dec. 6, 1978

192.09

WELI. PR-61-48-701

Owner: Lark in Frankl in

Screen: 1,210-1,250 feet

Elevation: 35 feet

Mar. 10, 1975

95.80

Mar. 17, 1976

97.52

Mar. 29, 1979

103.50

Mar. 31, 1980

100.85 
Table 2.--Water levels in observation wells in the study area, 1975-80--Continued

JEFFERSON COUNTY, TEXAS

$$
\begin{array}{ll}
\text { Water } \\
\text { Date }
\end{array}
$$

WELL PR-61-48-702

Owner: J. C. Chance

Screen: 448-468 feet

Elevation: 30 feet

$\begin{array}{ll}\text { Mar. 10, 1975 } & 40.88 \\ \text { Mar. 17, 1976 } & 40.75 \\ \text { Apr. 4, 1978 } & 42.98 \\ \text { Mar. 29, 1979 } & 43.92 \\ \text { Mar. 31, 1980 } & 43.21\end{array}$

WELL PR-62-17-902

Owner: W. S. Gillespie

Screen: $300-325$ feet

Elevation: 119 feet

Feb. 13, 1975

Dec. 8,1976

Dec. 7, 1977

32.45

Dec. 6,1978

32.93

WELL PR-62-33-211

Owner: Cougar Country Subdiv. Screen: 495-535 feet

Elevation: 68 feet

Mar. 25, 1975

Mar. 29, 1977

Apr. 5, 1978

69.4

Mar. 29, 1979

68.70

Apr. 8, 1980

70.14

WELL PR-62-33-401

Owner: City of Buna

Screen: 230-375 feet

Elevation: 72 feet

Mar. 24, 1975

31.70

Mar. 18, 1976

32.56

Mar. 29, 1977

32.46

Apr. 4, 1978

33.23

Mar. 29, 1979

35.24

Apr. 8, 1980

30.52

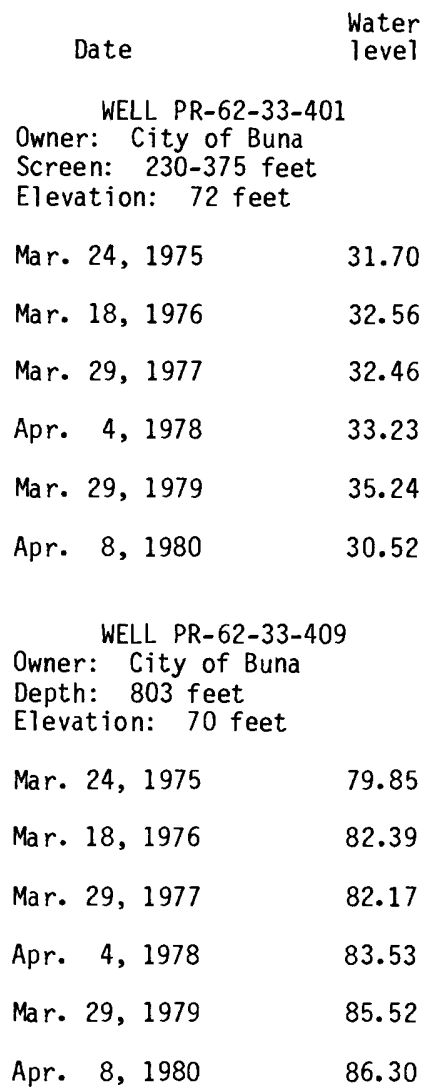

WELL PR-62-41-908

Owner: Farm Market

Depth: 70 feet

Elevation: 42 feet

Mar. 24, $1975 \quad 25.16$

Mar. 22, $1976 \quad 24.37$

Mar. 29, $1977 \quad 23.69$

Apr. 4, $1978 \quad 23.94$

Mar. 29, $1979 \quad 24.09$ $\begin{array}{ll}\text { Water } & \text { Wate } \\ \text { level }\end{array}$

WELL PT-61-64-502

Owner: Gulf States Util. Co. Screen: 306-435 feet

Elevation: 10 feet

Mar. 19, 1975

38.42

Mar. 19, 1976

81.88

Mar. 23, 1977

70.55

Apr. 6, 1978

34.19

May 2, 1979

35.52

WELL PT-61-64-509

Owner: Gulf States Util. Co. Screen: 380-542 feet Elevation: 8 feet

Mar. 19, 1975

34.81

Mar. 19, 1976

42.24

Mar. 23, 1977

41.25

Apr. 6, 1978

34.38

May 2,1979

35.58

WELL PT-61-64-902

Owner: Air Reduction Co. Screen: 497-550 feet Elevation: 20 feet

Mar. 19, 1975

45.10

Mar. 19, 1976

46.42

Mar. 23, 1977

45.61

Apr. 6, 1978

45.81

WELL PT-63-01-201

Owner: City of Groves Screen: $520-540$ feet

Elevation: 9 feet

Sept. 25, 1975

39.06

Mar. 19, 1976

40.42

Mar. 23, 1977

40.92

Apr. 6, 1978

41.02

May 1,1979

41.51 
Table 2.--Water levels in observation wells in the study area, 1975-80--Continued

NEWTON COUNTY, TEXAS

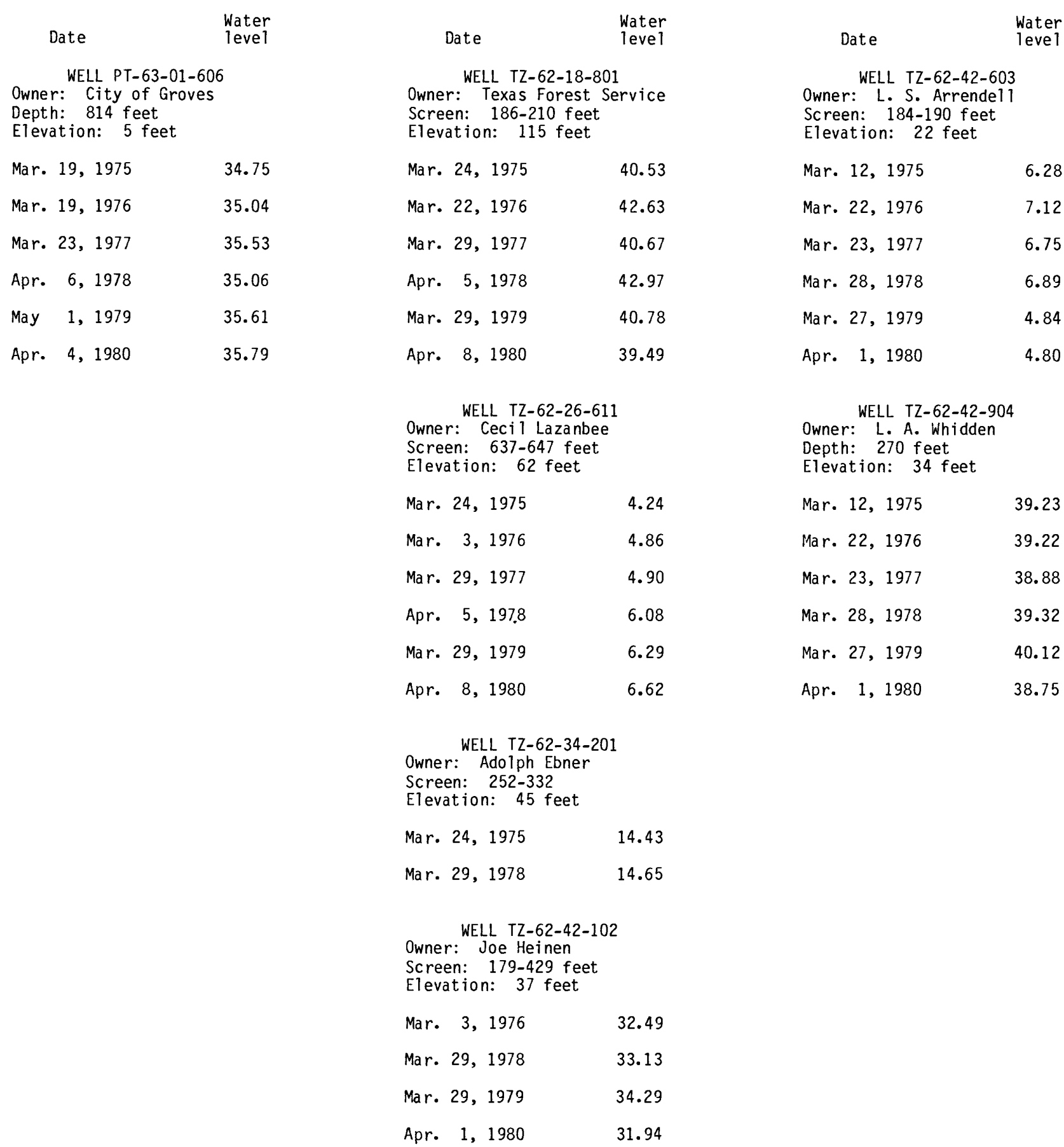


Table 2.--Water levels in observation wells in the study area, 1975-80--Continued

CALCASIEU PARISH, LOUISIANA

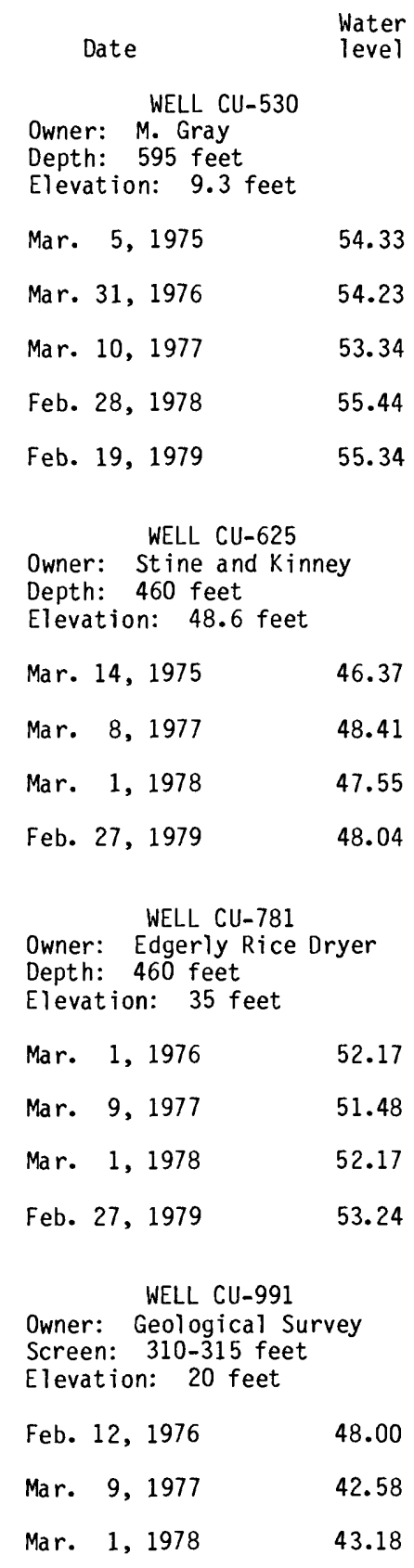

\section{CAMERON PARISH, LOUSIANA}

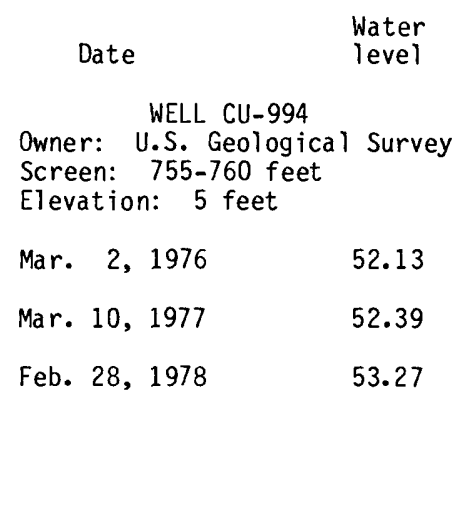

Water

Date

Teve

WELL CN-86L

Owner: U.S. Geological Survey Screen: 631-641 feet Elevaton: 3.66 feet

Mar. 5, 1975

49.85

Mar. 3, 1976

48.63

Feb. 23, 1977

48.82

Feb. 14, 1978

49.74

Feb. 28, 1979

50.14

Feb. 12, 1980

47.79

WELL CN-86U

Owner: U.S. Geological Survey

Screen: $525-535$ feet

Elevation: 3.66 feet

Mar. 5, 1975

49.71

Mar. 3, 1976

48.52

Feb. 23, 1977

48.67

Feb. 14, 1978

49.62

Feb. 28, 1979

50.16

Feb. 12, 1980

47.66

WELL CN-94

Owner: U.S. Geological Survey Screen: 1,112-1,118 feet

Elevation: 6.2 feet

Feb. 20, 1975

40.68

Mar. 2, 1976

41.38

Mar. 9, 1977

40.82

Mar. 28, 1978

41.02

Feb. 28,1979

41.80 


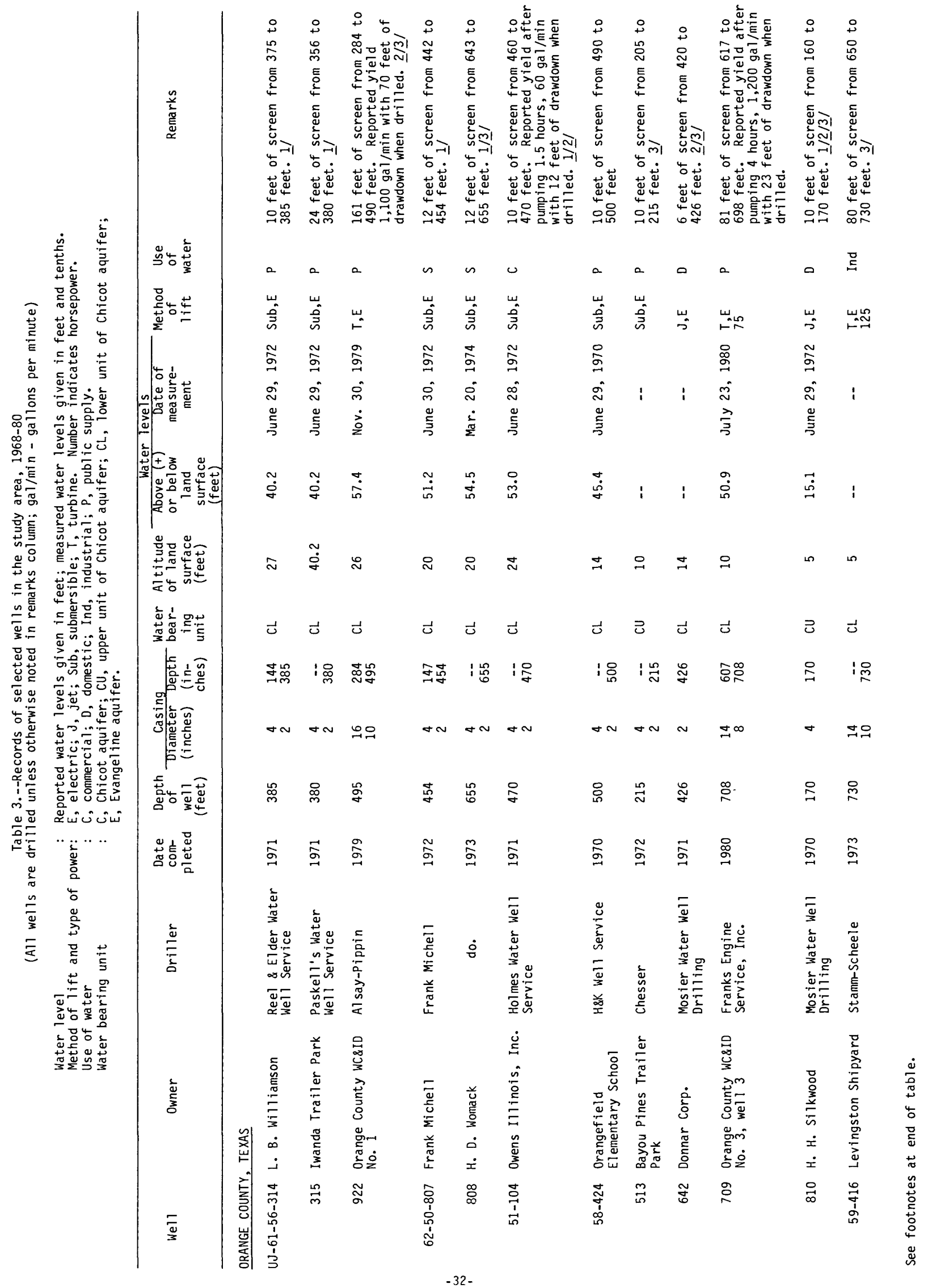




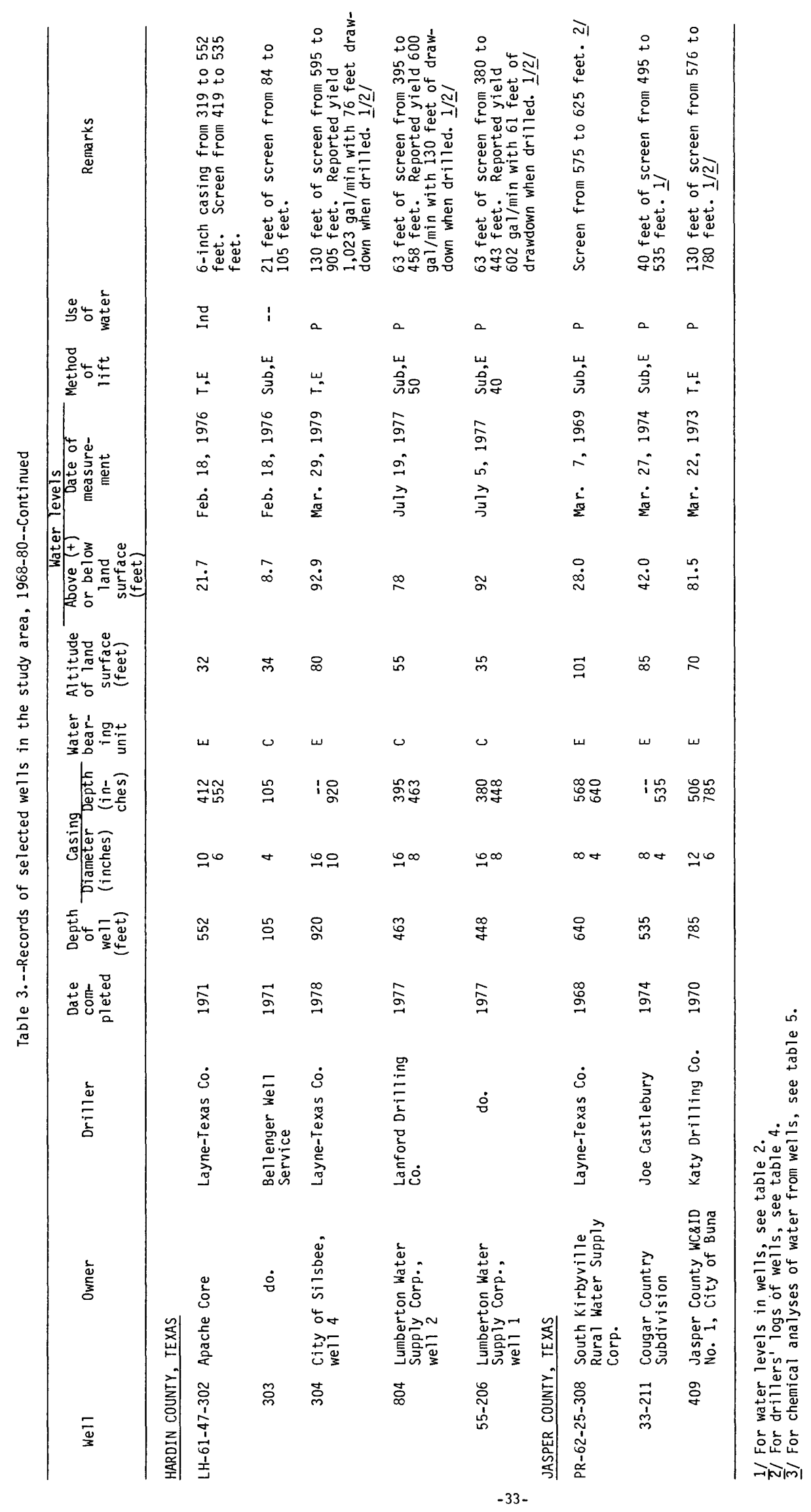


Table 4.--Drillers' logs of selected wells in the study area, 1968-80

ORANGE COUNTY, TEXAS

Thickness Depth

(feet) (feet)
WELL UJ-61-56-922

OWNER: Orange County WC\&ID No. 1 DRILLER: Alsay-Pippin Corp.
WELL UJ-62-58-642

OWNER: Donnar Corp.

DRILLER: Mosier Water Well Drilling

$\begin{array}{lrrl}\text { clay, sandy } & 15 & 15 & \text { topsoil } \\ \text { sandy } & 5 & 20 & \text { sand } \\ \text { shale } & 17 & 37 & \text { mud } \\ \text { sand } & 103 & 140 & \text { sand } \\ \text { shale } & 145 & 285 & \text { mud } \\ \text { sand } & 30 & 315 & \text { sand } \\ \text { shale } & 25 & 340 & \text { mud } \\ \text { sand } & 50 & 390 & \text { sand } \\ \text { shale } & 10 & 400 & \text { mud } \\ \text { sand } & 90 & 490 & \text { sand } \\ \text { shale } & 110 & 600 & \text { mud } \\ \text { shale, sandy } & 150 & 750 & \text { sand }\end{array}$

Thickness Depth

(feet) (feet)

WELL UJ-62-51-104

OWNER: Owens-Illinois, Inc. DRILLER: Holmes Water Well Service

\section{8}

8

$\begin{array}{llllllllllllll}13 & 21\end{array}$

61

$61 \quad 82$

$19-101$

5106

$56 \quad 162$

$8 \quad 170$

$14 \quad 184$

$76 \quad 260$

$4 \quad 264$

$120 \quad 384$

$42 \quad 426$

WELL UJ-62-58-810

OWNER: H. K. Silkwood DRILLER: Mosier Water Well Drilling

$\begin{array}{lrr}\text { clay } & 42 & 42 \\ \text { sand } & 59 & 101 \\ \text { clay } & 84 & 185 \\ \text { sand } & 5 & 190 \\ \text { clay } & 6 & 196 \\ \text { sand } & 11 & 207 \\ \text { clay } & 12 & 219 \\ \text { sand } & 16 & 235 \\ \text { clay } & 80 & 315 \\ \text { sand } & 50 & 365 \\ \text { clay } & 25 & 390 \\ \text { sand } & 80 & 470\end{array}$

mud

sand

mud

sand

mud

sand and rock

mud

sand
12

30

$17 \quad 59$

12

42

59

$7 \quad 66$

268

$39 \quad 107$

$15 \quad 122$

$3 \quad 125$

$23 \quad 148$

$22 \quad 170$ 
Table 4.--Drillers' logs of selected wells in the study area, 1968-80--Continued

\section{HARDIN COUNTY, TEXAS}

Thickness Depth

(feet) (feet)

WELL LH-61-47-304

OWNER: City of Silsbee, well 4

DRILLER: Layne-Texas Co.

\begin{tabular}{|c|c|c|}
\hline topsoil & 4 & 4 \\
\hline clay & 42 & 46 \\
\hline sand & 275 & 321 \\
\hline shale, sandy & 20 & 341 \\
\hline sand & 36 & 377 \\
\hline shale, sandy & 41 & 418 \\
\hline sand & 50 & 468 \\
\hline shale & 24 & 492 \\
\hline shale, sandy & 70 & 562 \\
\hline sand (broken) & 49 & 611 \\
\hline shale & 98 & 709 \\
\hline sand & 61 & 770 \\
\hline shale & 10 & 780 \\
\hline sand & 11 & 791 \\
\hline shale & 11 & 802 \\
\hline shale, sandy & 13 & 815 \\
\hline sand & 27 & 842 \\
\hline shale & 55 & 897 \\
\hline sand and shale & 39 & 936 \\
\hline shale & 50 & 986 \\
\hline sand and shale & 62 & 1,048 \\
\hline shale and sand streaks & 52 & 1,100 \\
\hline
\end{tabular}

Thickness Depth (feet) (feet)

WELL LH-61-47-804

OWNER: Lumberton Water Supply Corp. DRILLER: Lanford Drilling Co.

\begin{tabular}{|c|c|c|}
\hline clay and sand & 2 & 2 \\
\hline clay & 6 & 8 \\
\hline sand & 20 & 28 \\
\hline sand and clay streaks & 87 & 115 \\
\hline sand & 95 & 210 \\
\hline clay & 8 & 218 \\
\hline sand & 62 & 280 \\
\hline clay and shale & 50 & 330 \\
\hline sand & 40 & 370 \\
\hline shale & 15 & 385 \\
\hline shale, sandy & 10 & 395 \\
\hline sand & 70 & 465 \\
\hline shale & 10 & 475 \\
\hline sand & 45 & 520 \\
\hline shale & 5 & 525 \\
\hline sand & 35 & 560 \\
\hline shale and sand & 30 & 590 \\
\hline sand & 10 & 600 \\
\hline shale, sandy, and sand streaks & 100 & 700 \\
\hline shale & 30 & 730 \\
\hline shale, sandy, and sand & 50 & 780 \\
\hline
\end{tabular}


Table 4.--Drillers' logs of selected wells in the study area, 1968-80--Continued

Thickness Depth

(feet) (feet)

WELL LH-61-55-206

OWNER: Lumberton Water Supply Corp.

DRILLER: Lanford Drilling Co.

\begin{tabular}{|c|c|}
\hline sand & 2 \\
\hline clay & 5 \\
\hline sand & 13 \\
\hline clay & 90 \\
\hline sand & 40 \\
\hline clay & 10 \\
\hline sand & 50 \\
\hline shale & 40 \\
\hline sand & 20 \\
\hline shale & 30 \\
\hline sand, brown, fine & 30 \\
\hline shale & 50 \\
\hline sand, white, coarse & 70 \\
\hline shale & 5 \\
\hline shale, sandy, and sand streaks & 75 \\
\hline shale & 40 \\
\hline shale, sandy & 100 \\
\hline shale & 40 \\
\hline $\begin{array}{l}\text { sand, sandy shale, and sand } \\
\text { streaks }\end{array}$ & 63 \\
\hline
\end{tabular}


Table 4.--Drillers' logs of selected wells in the study area, 1968-80--Continued

\section{JASPER COUNTY, TEXAS}

Thickness Depth

(feet) (feet)

WELL PR-62-25-308

OWNER: South Kirbyville Rural Water Supply Corp. DRILLER: Layne-Texas Co.

\begin{tabular}{|c|c|}
\hline topsoil & 4 \\
\hline clay & 42 \\
\hline sand & 275 \\
\hline shale, sandy & 20 \\
\hline sand & 36 \\
\hline shale, sandy & 41 \\
\hline sand & 50 \\
\hline shale & 24 \\
\hline shale, sandy & 70 \\
\hline sand (broken) & 49 \\
\hline shale & 98 \\
\hline sand & 61 \\
\hline shale & 10 \\
\hline sand & 11 \\
\hline shale & 11 \\
\hline shale, sandy & 13 \\
\hline sand & 27 \\
\hline shale & 55 \\
\hline sand and shale & 39 \\
\hline shale & 50 \\
\hline sand and shale & 62 \\
\hline shale and sand streaks & 52 \\
\hline
\end{tabular}
6 41 77 18 468
Thickness Depth

(feet) (feet)

WELL PR-62-33-409

OWNER: Jasper County WC\&ID No. 1, City of Buna DRILLER: Katy Drilling Co.

$\begin{array}{lrr}\text { clay } & 10 & 10 \\ \text { sand } & 5 & 15 \\ \text { clay } & 25 & 40 \\ \text { sand, clay streaks } & 170 & 210 \\ \text { clay, sand streaks } & 70 & 280 \\ \text { sand, thin clay streaks } & 85 & 365 \\ \text { tree } & 10 & 375 \\ \text { clay, hard } & 82 & 457 \\ \text { sand } & 42 & 499 \\ \text { shale, hard } & 13 & 512 \\ \text { sand } & 46 & 558 \\ \text { shale } & 10 & 568 \\ \text { sand } & 11 & 579 \\ \text { shale, hard } & 51 & 630 \\ \text { shale, sandy, sand streaks } & 87 & 717 \\ \text { sand, thin streaks clay } & 28 & 745 \\ \text { clay } & 37 & 748 \\ \text { sand } & 18 & 785 \\ \text { sand streaks and clay } & & 803\end{array}$

clay 10 21 


\begin{tabular}{|c|c|c|c|c|c|c|c|c|c|c|c|}
\hline & 产离岩崖递 & $\begin{array}{llll}1 & 1 & 1 & 1\end{array}$ & 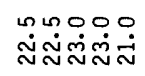 & $: 1: 1: 1$ & 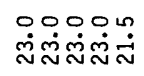 & $\mathrm{i}$ & : $1: 1:$ & i: $1: 1:$ i & i: & : : : : : & 1: $: 1:$ i \\
\hline & I⿳亠幺幺̆丶 & 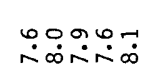 & 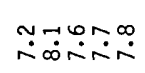 & 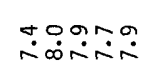 & 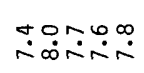 & $\stackrel{\dddot{2}}{\sim}$ & 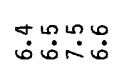 & mårín & $\stackrel{+}{\sim} \stackrel{0}{\infty}$ & 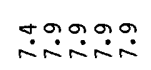 & 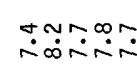 \\
\hline & 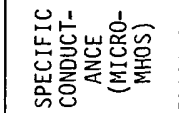 & 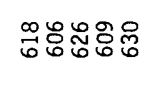 & 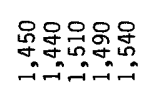 & 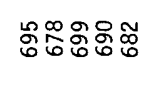 & 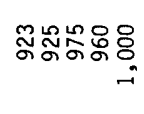 & $\stackrel{\infty}{\vec{\sigma}}$ & 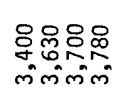 & 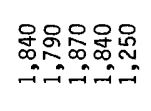 & 兽导 & 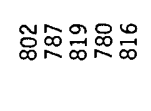 & 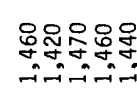 \\
\hline & 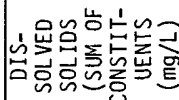 & : : $1: 1:$ i & $1: 1: 1$ & 1：１：：： & $\begin{array}{lllll}1 & 1 & 1 & 1 & 1\end{array}$ & $\underset{\sigma}{\vec{\sigma}}$ & $1: 1: 11$ & $1: 1: 1$ & i : & 1: $1: 11$ & $\begin{array}{llll}1 & 1 & 1 & 1\end{array}$ \\
\hline $\begin{array}{l}\bar{n} \\
\text { ò } \\
\text { in }\end{array}$ & 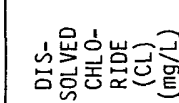 & 조ㅅㅗㅗ요요 & 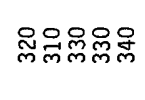 & 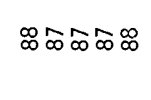 & 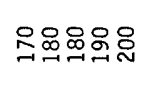 & ณ & 양요융 & 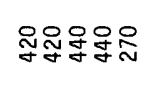 & ర్లింి & 品昌昌昌昌 & 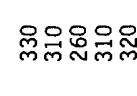 \\
\hline 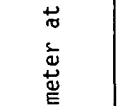 & 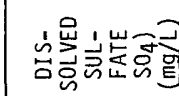 & $\stackrel{\infty}{0}_{0}^{\infty}$ & $\dddot{0}$ & • & $\dddot{0}_{00}+\stackrel{\circ}{\circ}$ & $i$ & 品 & $000 \stackrel{+m}{m}$ & 00 & $0^{+\square+\tilde{N}}$ & 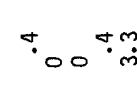 \\
\hline : & 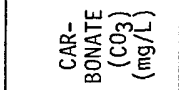 & 00000 & 00000 & 00000 & 00000 & 0 & 0000 & 00000 & 00 & 00000 & 00000 \\
\hline 吾 & 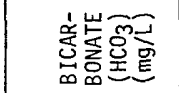 & 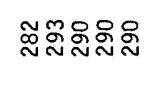 & 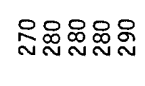 & 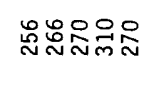 & 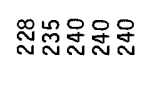 & స్ & 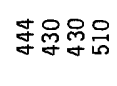 & జ & ลีని & 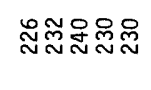 & : \\
\hline 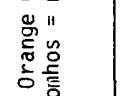 & 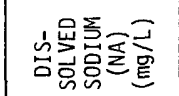 & : : : : : : & i $1: 11:$ & 1: $1: 1:$ & 1: : : : i & i & $1: 1: 1$ & : $1: 1: 1:$ & i i & 1：：１：： & 1:1:1: \\
\hline 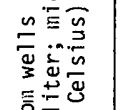 & 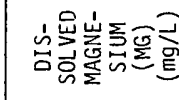 & : $: 1: 1: 1$ & : $1: 1: 11$ & : $1: 1: 1: 11$ & $1: 1: 11$ & $\stackrel{\Upsilon}{-}$ & $:: 1: 1$ & $1: 1: 1$ & : : & : $1: 11:$ & 1:11: \\
\hline 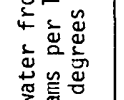 & 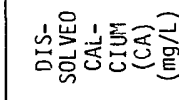 & $1: 1: 1: 1$ & $1: 1: 1$ & i: $1: i:$ & $1: 1: 1$ & $\vec{b}$ & 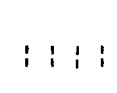 & $1: 1: 1$ & i i & : $: 1: 1: 1$ & $1: 1111$ \\
\hline 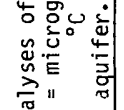 & 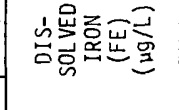 & $1: 1: 1:$ & $\begin{array}{lllll}1 & 1 & 1 & 1 & 1\end{array}$ & $1: 1: 11$ & $1: 1: 1$ & $\overrightarrow{0}$ & $1: 1: 1:$ & : : : : : & $1:$ & ：：１：１ & : : $1: 1:$ \\
\hline 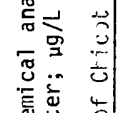 & 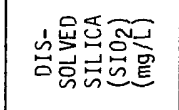 & $1: 1: i$ & $1: 11:$ & 1: $11: 1$ & 1: $14: 11$ & $\stackrel{\circ}{\circ}$ & 1：：１ & 1：１：１： & i i & : $: 1: 1: 1$ & $1: 1: 1$ \\
\hline 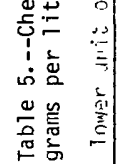 & 崖 & 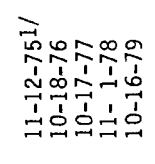 & 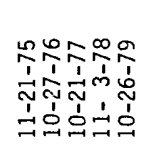 & 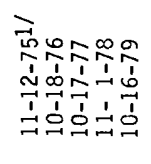 & 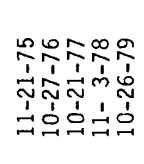 & 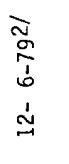 & 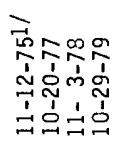 & 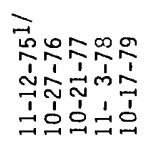 & 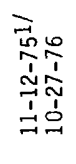 & 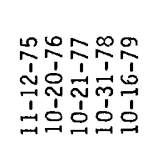 & 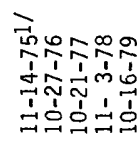 \\
\hline 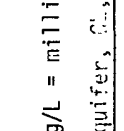 & 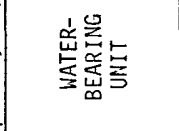 & 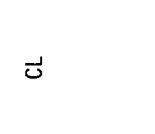 & $\overleftrightarrow{\circlearrowleft}$ & $\breve{\circlearrowleft}$ & $\breve{U}$ & 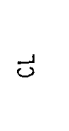 & $\Xi$ & $\vec{u}$ & $\vec{\jmath}$ & $\vec{\jmath}$ & $\breve{\circlearrowleft}$ \\
\hline 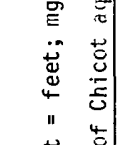 & 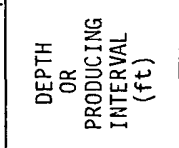 & 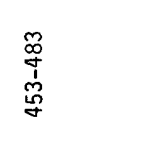 & 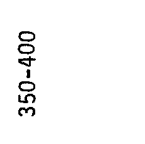 & 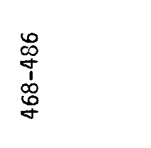 & 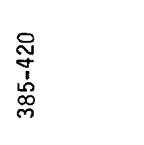 & 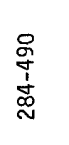 & $\begin{array}{l}0 \\
0 \\
\vdots \\
\vdots \\
\vdots\end{array}$ & $\bar{\Xi}$ & 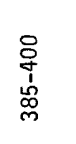 & 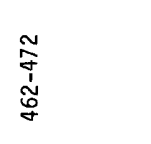 & $\begin{array}{l}\text { 号 } \\
\stackrel{\tilde{N}}{\hat{n}}\end{array}$ \\
\hline 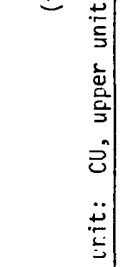 & 㞻 & 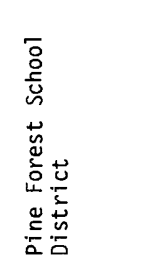 & 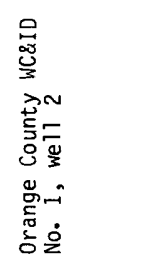 & 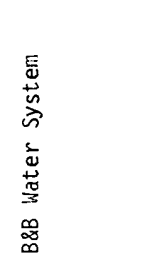 & 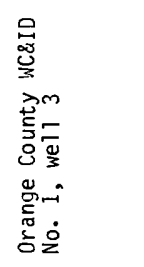 & 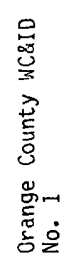 & 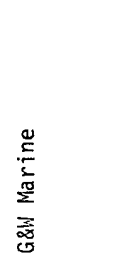 & 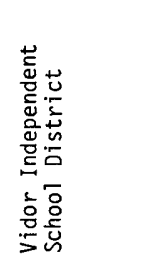 & $\begin{array}{l}\dot{\Xi} \\
\vdots \\
\vdots \\
\delta\end{array}$ & 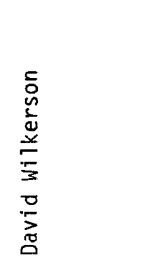 & 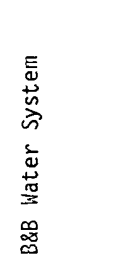 \\
\hline 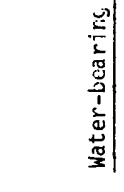 & 岀 & 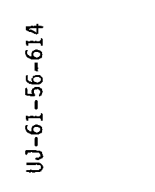 & Б̊ & $\vec{\sigma}$ & के & న్ & $\begin{array}{l}\tilde{O} \\
\frac{1}{1} \\
0\end{array}$ & జ్ల & 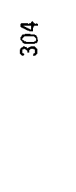 & 号 & 。্ \\
\hline
\end{tabular}




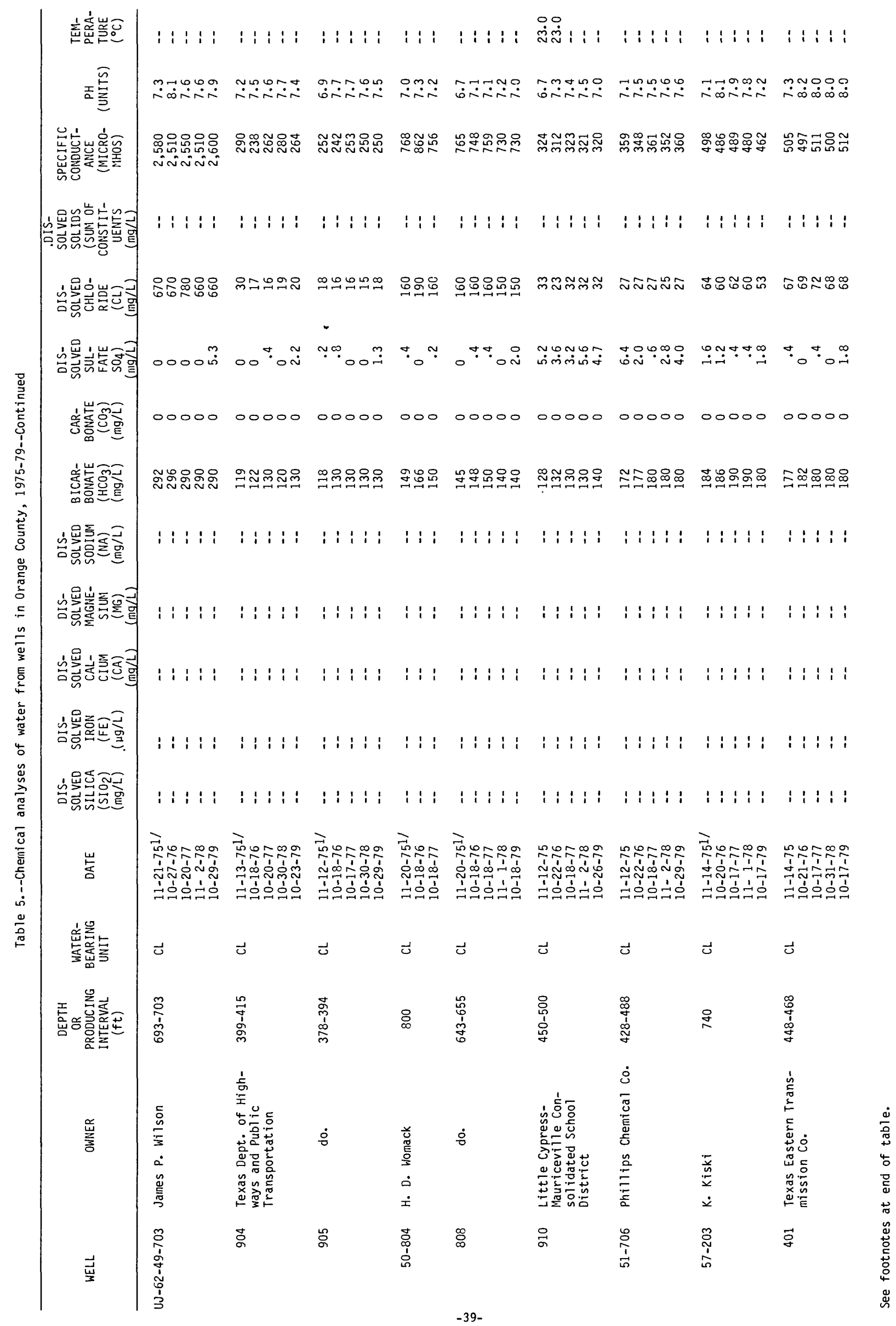




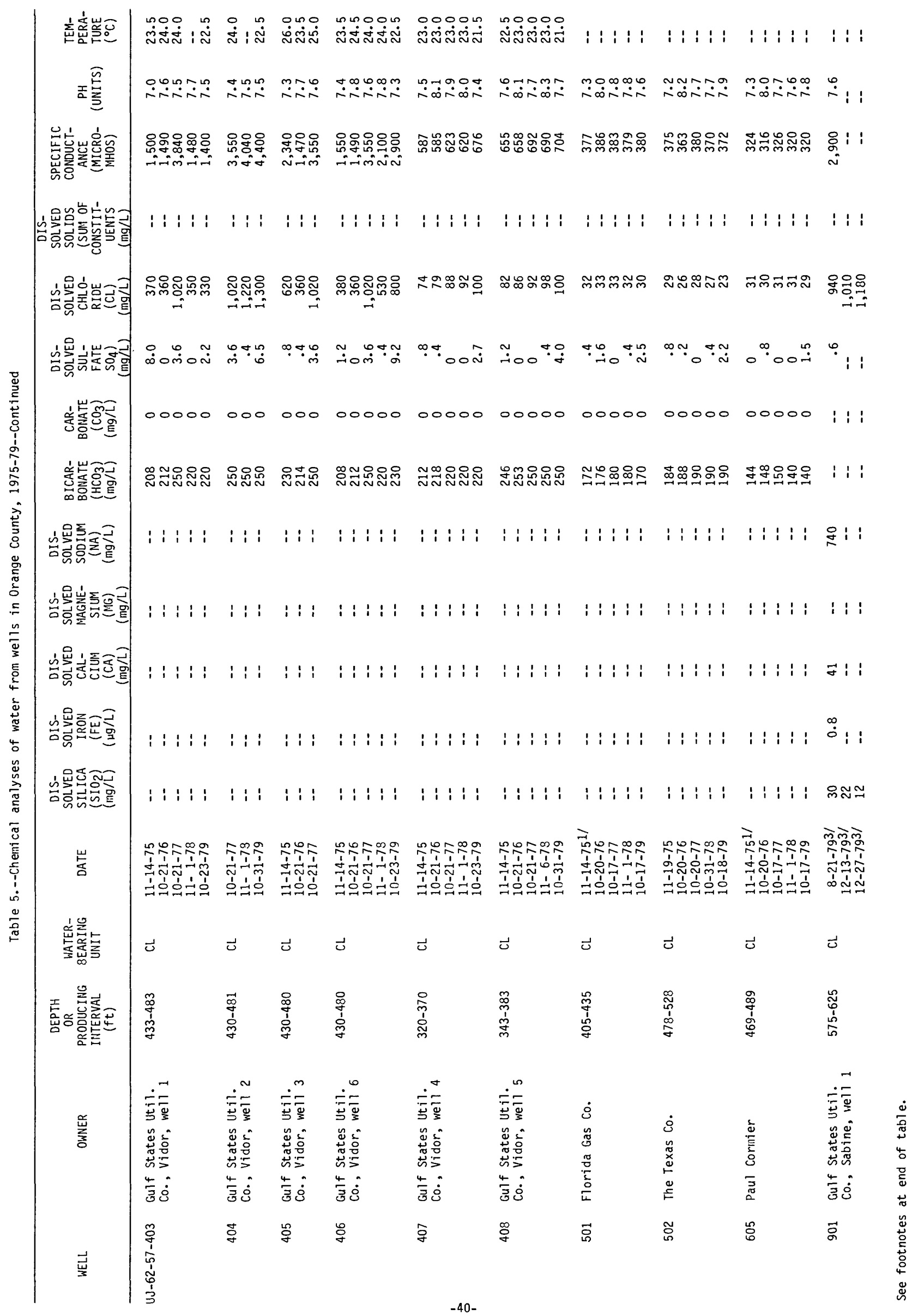




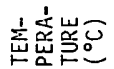

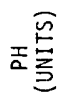

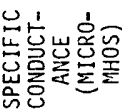

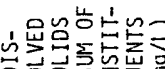

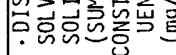

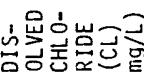

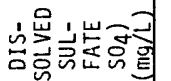

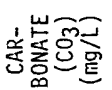

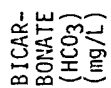

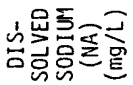

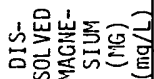

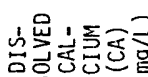

.

a

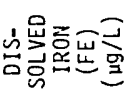

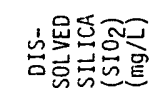

:

崖

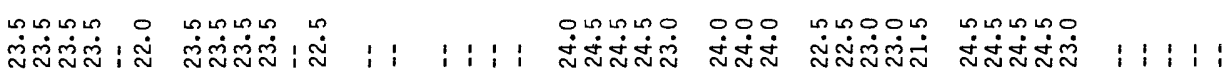

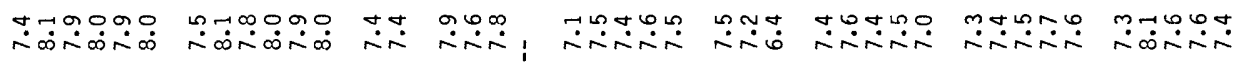

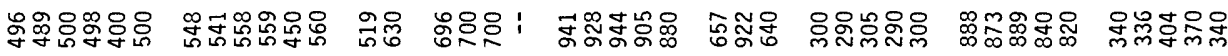

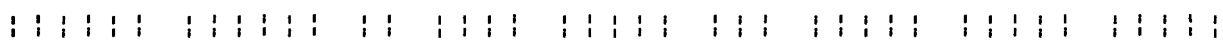

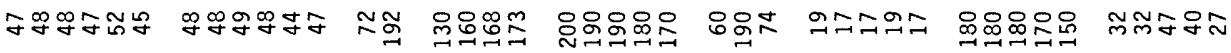

$\dddot{m}_{0}$

$0000,00000,00 ; 00: 1,00000000000000000000000$

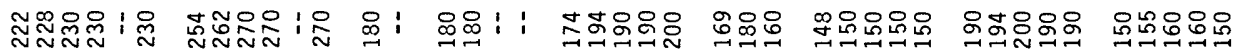

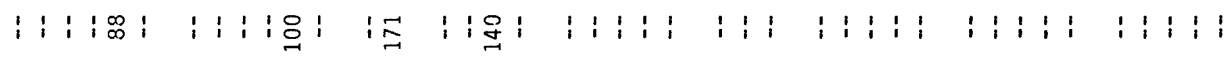

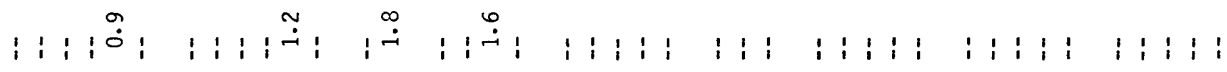

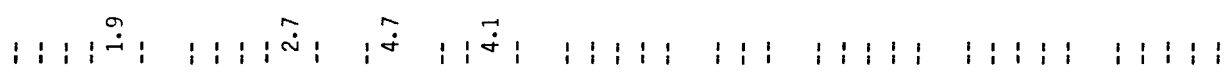

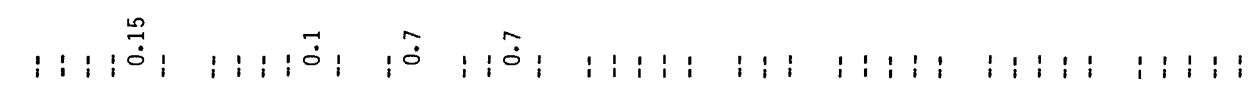

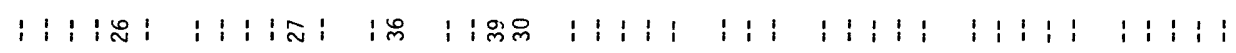

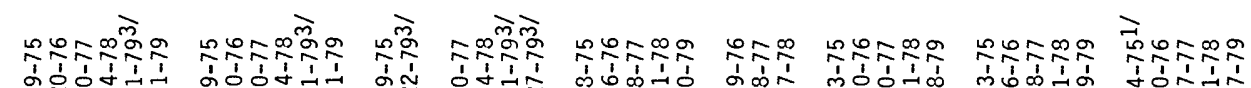

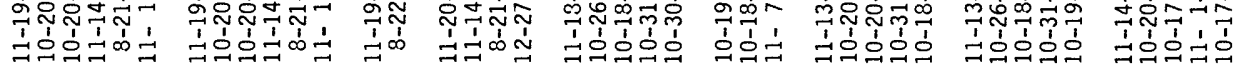

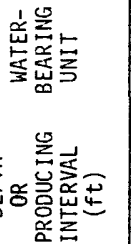

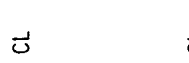

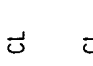

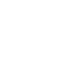

芩

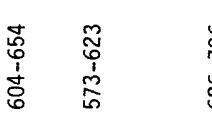

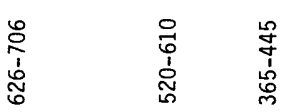

$\begin{array}{ll}0 & 0 \\ 0 & 0 \\ 0 & 0 \\ 0 & 0 \\ 0 & 0\end{array}$

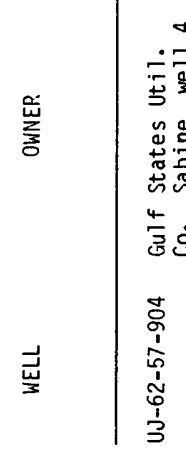

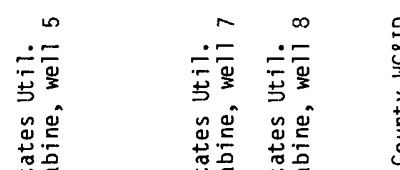

ठำ

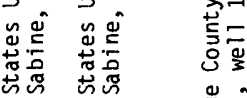

苋

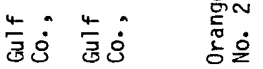

$t_{0} \quad 4_{0}^{-}$

ज請

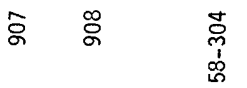

号 胥

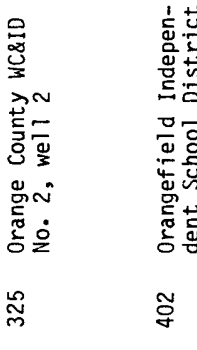

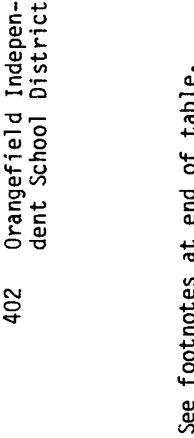




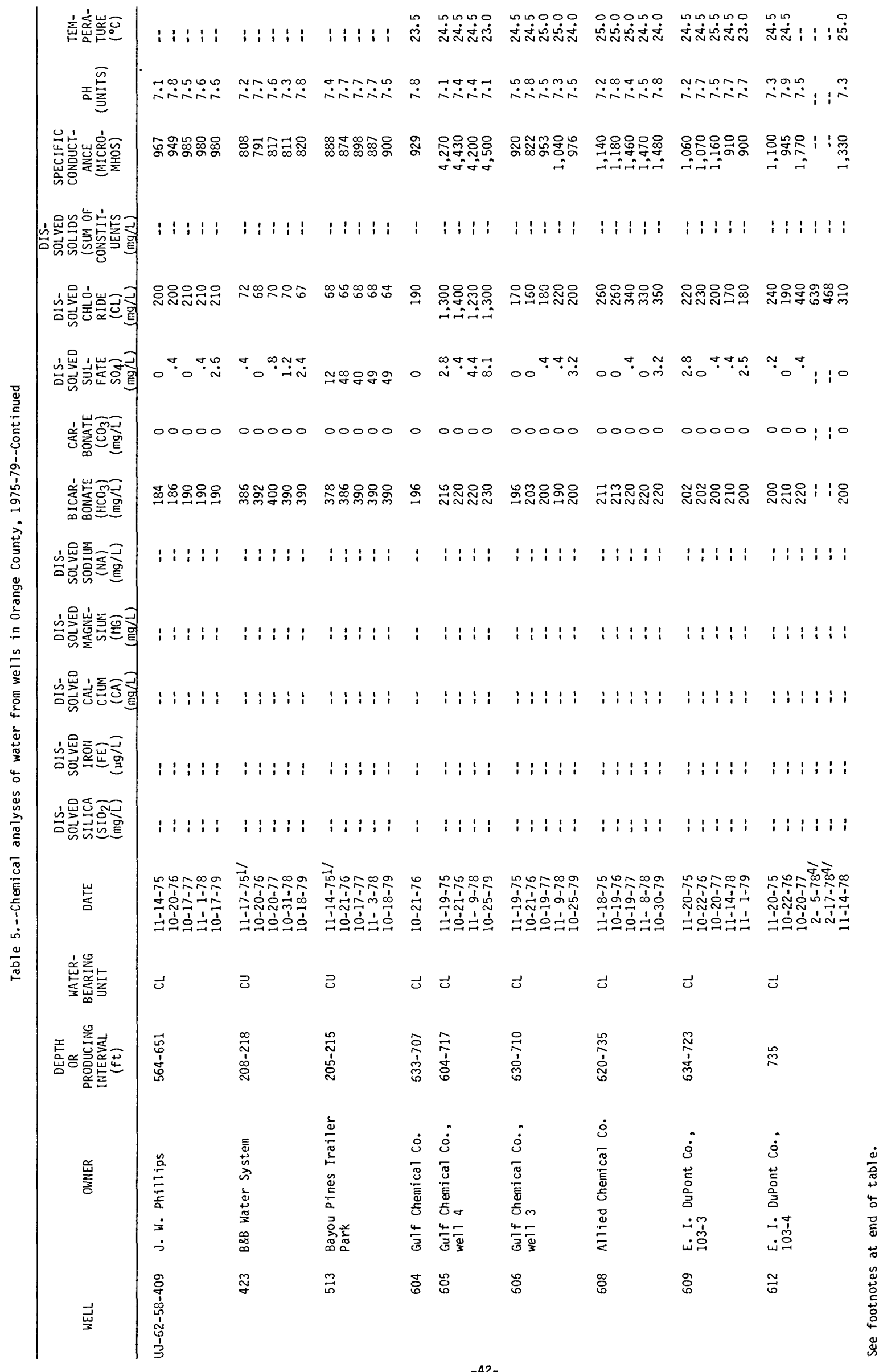




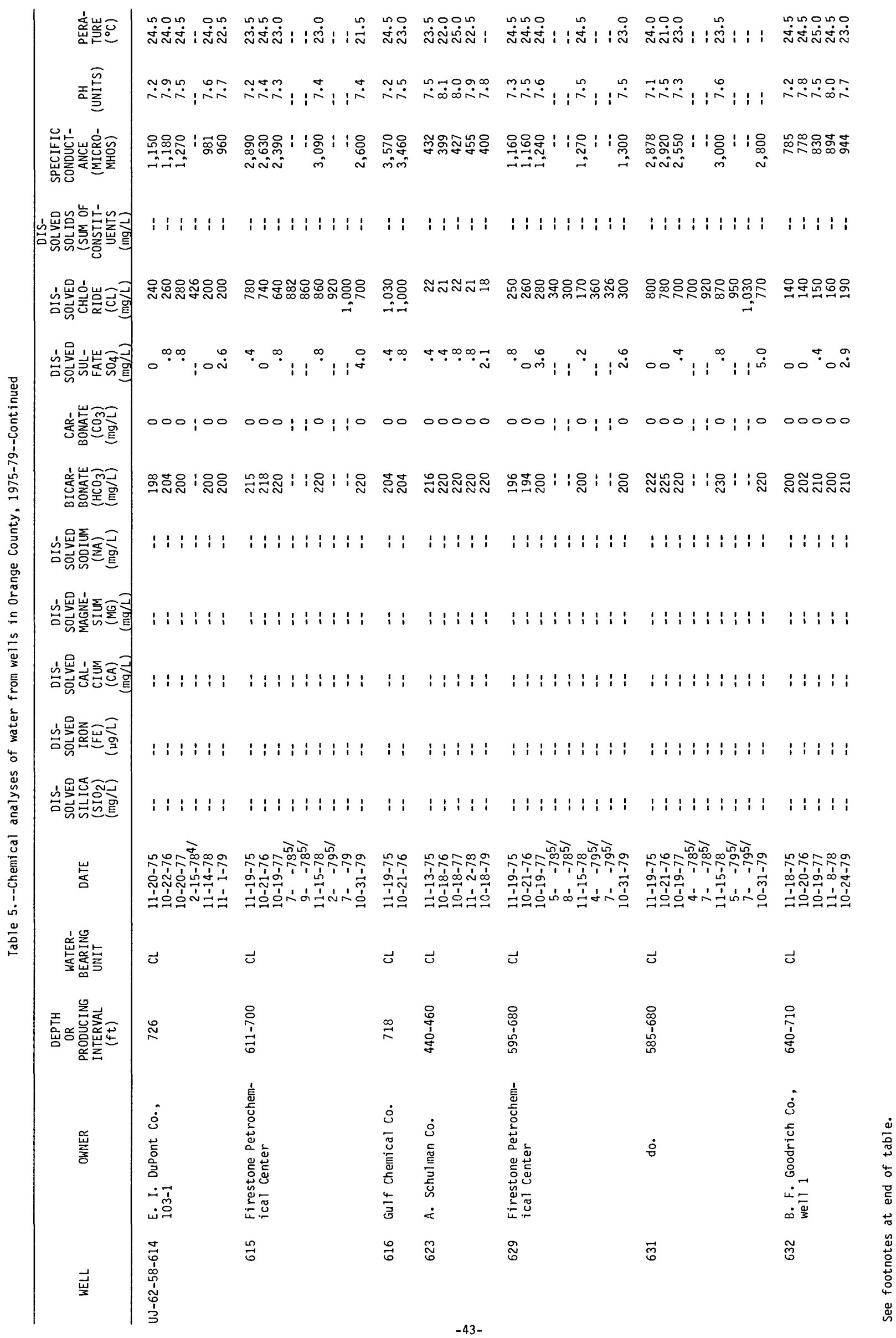




\begin{tabular}{|c|c|c|c|c|c|c|c|c|}
\hline 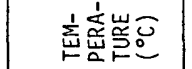 & 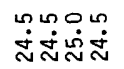 & 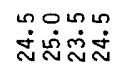 & 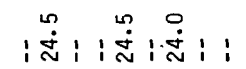 & 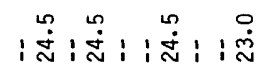 & 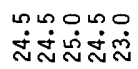 & $1: \stackrel{0}{\infty}$ & $:: 1: i$ & 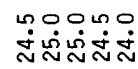 \\
\hline$\Sigma \hat{气}$ & 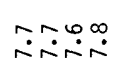 & 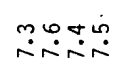 & 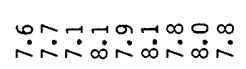 & 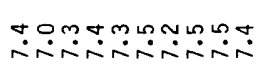 & 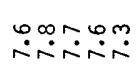 & 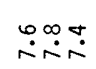 & 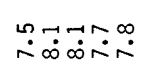 & 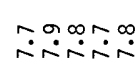 \\
\hline 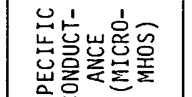 & 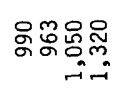 & 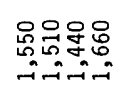 & 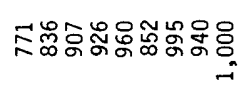 & 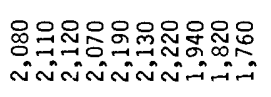 & 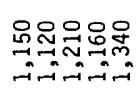 & 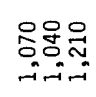 & 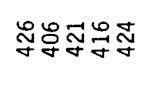 & 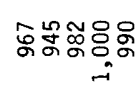 \\
\hline 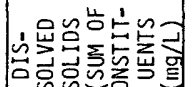 & $1: i:$ & $1: 1: 1:$ & 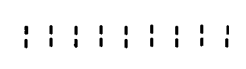 & 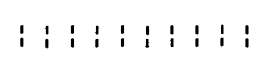 & $\begin{array}{llll}1 & 1 & 1 & 1\end{array}$ & $1: 1$ & : : : : : & 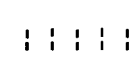 \\
\hline 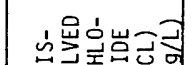 & ్ำ్సిని & 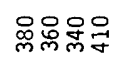 & 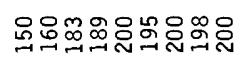 & 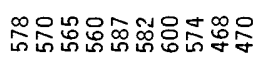 & 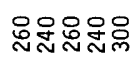 & సิని:్ల & 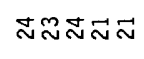 & ి్సిర్సి్ํํํ \\
\hline 岕岂崖司司 & $00^{+0}$ & $000^{\circ}$ & $\stackrel{\circ}{\text { i. }} 0000000 \stackrel{\sim}{m}$ & $000 \stackrel{0}{100000 \stackrel{+}{q}}$ & $0000 \stackrel{\infty}{1}$ & ○: & $00 \%$ تี & م0 \\
\hline & 0000 & 0000 & 000000000 & 0000000000 & 00000 & 000 & 00000 & 00000 \\
\hline & 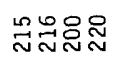 & 퓨유 & 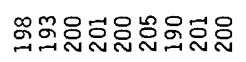 & 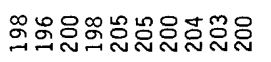 & สีสลิ & 뉴ํำ & స్సిన్లిల్లిల్లి & 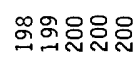 \\
\hline & $:: 1: i$ & $\begin{array}{l}1 \\
1\end{array}: 1: i$ & $1: 1: 1: 1: 1: 1:$ & $1: 1: 1: 1: 1: 1: 1$ & 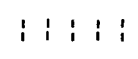 & $1: 1$ & $\begin{array}{lllll} & 1 & 1 & 1 & 1\end{array}$ & $1: 1: 1$ \\
\hline 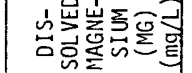 & i $: 1: 1$ & 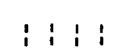 & $m ! \sim m ! m ! m !$ & 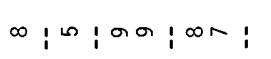 & 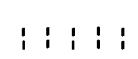 & $1: 1$ & i $: 1: 1:$ & $: \mathfrak{i}: 1:$ : \\
\hline 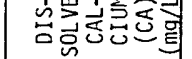 & $1: 1: 1$ & $1: 1:$ & $0: \infty, \infty ; 0$ ! & 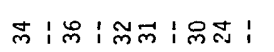 & : $1: 1: 1$ & i : i & : $1: 1: 1$ & $1: 1: 1:$ \\
\hline & $: 1: 1:$ & $: 1: 1:$ & $:: 1: 1: 1:\{$ & 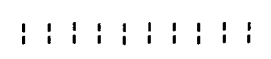 & $:: 1: i$ & $1: 1$ & $1: 1: 1$ & $: 1: 1:$ \\
\hline 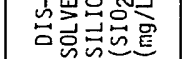 & $1: 1: 1$ & $\begin{array}{lll}1 & 1 & 1\end{array}$ & 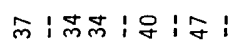 & 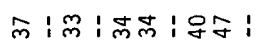 & : $: 1: 1:$ & $1: i$ & : $1: 1: 1$ & 1: $1: 1:$ \\
\hline 訔 & 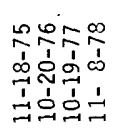 & 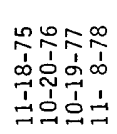 & 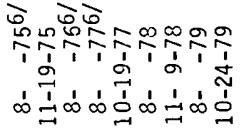 & 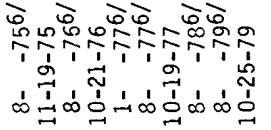 & 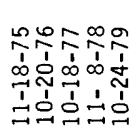 & 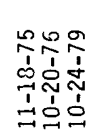 & 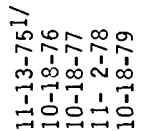 & 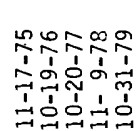 \\
\hline 岕氮氙 & $\vec{d}$ & $\vec{\circlearrowleft}$ & $\breve{\omega}$ & $\breve{\circlearrowleft}$ & 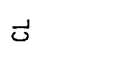 & 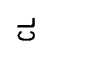 & $\breve{\circlearrowleft}$ & $\vec{\circlearrowleft}$ \\
\hline 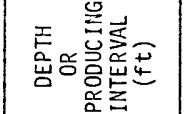 & 芯 & 兑 & 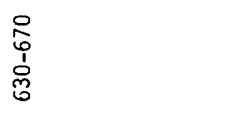 & 兽 & 芯 & 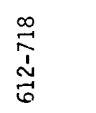 & 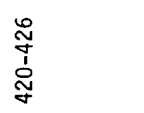 & $\stackrel{\square}{\circ}$ \\
\hline 䒿 & 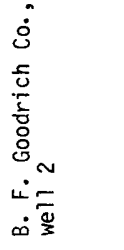 & 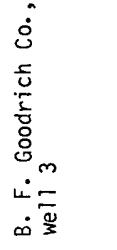 & 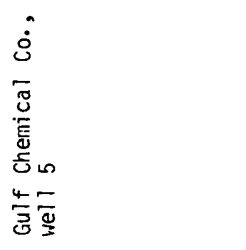 & 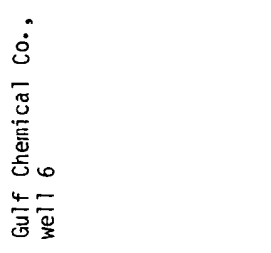 & 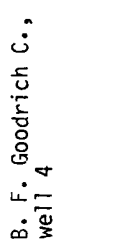 & 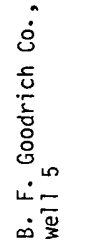 & 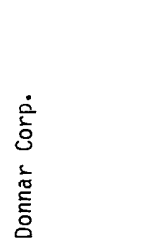 & 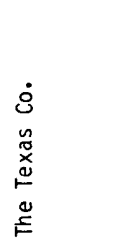 \\
\hline 岁 & 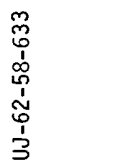 & 苞 & $\hat{\mathfrak{b}}$ & $\stackrel{\infty}{0}$ & 今్ & 惫 & $\frac{y}{6}$ & $\stackrel{5}{i}$ \\
\hline
\end{tabular}




\begin{tabular}{|c|c|c|c|c|c|c|c|c|c|c|}
\hline 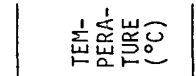 & 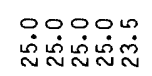 & 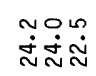 & 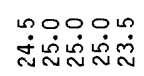 & : : : : : & $\begin{array}{l}00000 \\
\dot{\sim} \dot{N} \dot{\sim} \dot{N}\end{array}$ & $\begin{array}{l}0: 0 \\
\dot{\sim} \dot{\sim} \\
\tilde{N}\end{array}$ & 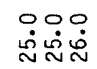 & : : & 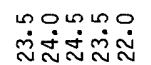 & 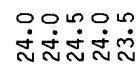 \\
\hline 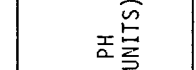 & 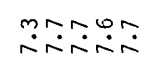 & $\stackrel{2}{\therefore}=0$ & 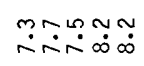 & 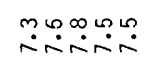 & 苗 & $\because 2$ & 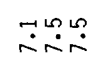 & $\stackrel{m}{\sim}$ & 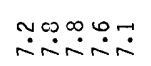 & 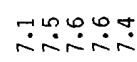 \\
\hline 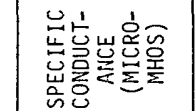 & 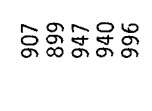 & 용용용ㅁㅇ & 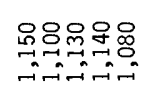 & 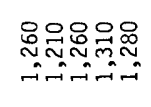 & 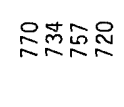 & fog & 密总品 & 봉응 & 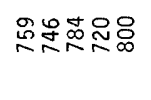 & 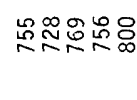 \\
\hline 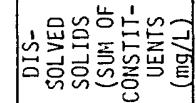 & $1: i: 1: i$ & $1: 1$ & $1: 11:$ & 1: i i i i & 1 $1: 1:$ & : : & $1: 11$ & i : & 111111 & 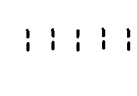 \\
\hline 空芦定 & 옴옴엄엄염 & ₹®్m & 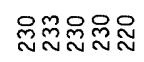 & 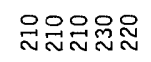 & 守守品品 & ถ⿻ำ & \& $\infty \infty$ & 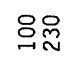 & 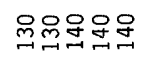 & 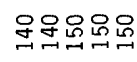 \\
\hline 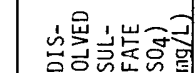 & $000 \stackrel{+\pi}{\sim}$ & $\stackrel{+m}{\sim}$ & †. & † & مُ بـ & $\stackrel{\infty}{\stackrel{\infty}{\sim}}$ & . & \%。 & $\because \stackrel{\infty}{\circ} \circ \stackrel{m}{m}^{2}$ & تُمْ \\
\hline & 00000 & 000 & 00000 & 00000 & 0000 & 00 & 000 & 00 & 00000 & 00000 \\
\hline 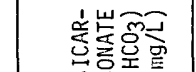 & న్సిన్లిన్లి & సిని స్ & 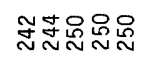 & 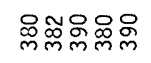 & సపః心 & 禺禺 & సేస్ & 윰요 & تีసีని & 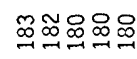 \\
\hline 岂岂总言 & $\begin{array}{lllll}1 & 1 & 1 & 1 & 1\end{array}$ & $1: 1$ & : $1: i: 1$ & : $1: 1: 1:$ & : $1: 1:$ & : : & i : $1:$ & : : & : : : : : : & 1: $1: 1$ \\
\hline 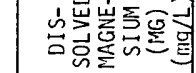 & $\begin{array}{lllll}1 & 1 & 1 & 1 & 1\end{array}$ & $1: 11$ & 1: $1: 11:$ & $\begin{array}{lllll}1 & 1 & 1 & 1 & 1\end{array}$ & $\begin{array}{llll}1 & 1 & 1 & 1\end{array}$ & $1:$ & $1: 1:$ & i i & : : : : : : & 1:1: \\
\hline 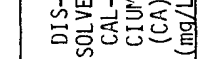 & : : : : : 1 & i : i & : $1: 1: 11$ & $1: 1: 11$ & $1: 1: 1:$ & : : & $1: 1:$ & : : & : : : : : : & 1:1: \\
\hline 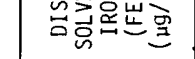 & : $1: 1:$ i & $1: i$ & $1: 111$ & : : :1: & i i: i & : i & $i: i$ & i i & $1: 1: i$ & i: $1: 1 ;$ \\
\hline 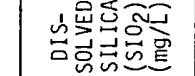 & : $1: 1: i$ & $1: 1:$ & $\begin{array}{lllll}1 & 1 & 1 & 1 & 1\end{array}$ & : $1: 1: i$ & : : : : i & : : & $1: 1$ & : i & i : i : i & 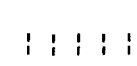 \\
\hline 峪 & 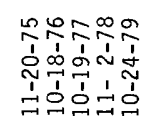 & 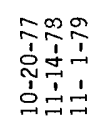 & 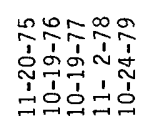 & 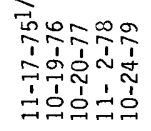 & 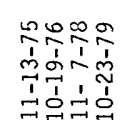 & 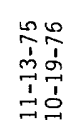 & 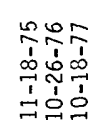 & 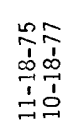 & 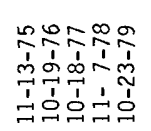 & 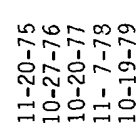 \\
\hline 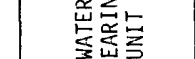 & $\vec{\circlearrowleft}$ & $\vec{\circlearrowright}$ & $\vec{\circlearrowright}$ & 己 & $\vec{\circlearrowright}$ & $\vec{\circlearrowleft}$ & $\vec{U}$ & $\vec{\circlearrowleft}$ & $\vec{\circlearrowleft}$ & $\vec{d}$ \\
\hline 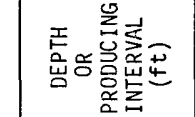 & $\begin{array}{l}\text { N } \\
\hat{0} \\
\vdots \\
0 \\
0\end{array}$ & $\underset{8}{\mathscr{O}}$ & $\begin{array}{l}0 \\
0.0 \\
1 \\
0 \\
0\end{array}$ & $\begin{array}{l}\stackrel{0}{1} \\
\stackrel{1}{0} \\
\stackrel{0}{-1}\end{array}$ & $\begin{array}{l}: 0 \\
0.0 \\
1 \\
0 \\
0 \\
0\end{array}$ & 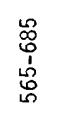 & $\stackrel{g}{1}$ & 号 & 氶 & $\begin{array}{l}\text { 옹 } \\
\text { ì } \\
\text { on }\end{array}$ \\
\hline 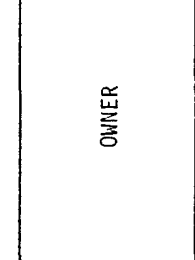 & 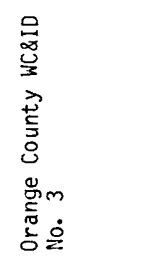 & 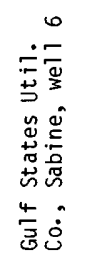 & 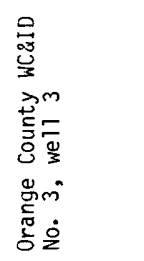 & 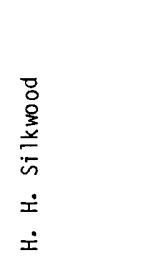 & 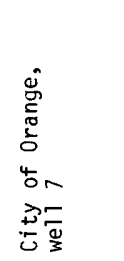 & 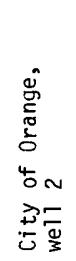 & 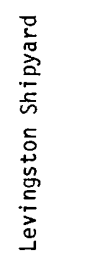 & $\dot{\theta}$ & 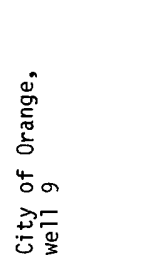 & 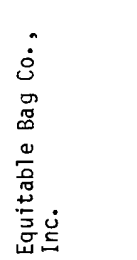 \\
\hline 岁 & 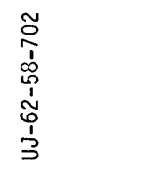 & $\stackrel{\infty}{\circ}$ & 今े & $\underset{\infty}{\stackrel{P}{\infty}}$ & $\begin{array}{l}\overrightarrow{0} \\
\overrightarrow{1} \\
0\end{array}$ & $\stackrel{m}{\rightarrow}$ & 吉 & $\stackrel{\circ}{\circ}$ & $\underset{\mathbb{N}}{\mathbb{N}}$ & $\underset{త}{\mathbb{N}}$ \\
\hline
\end{tabular}




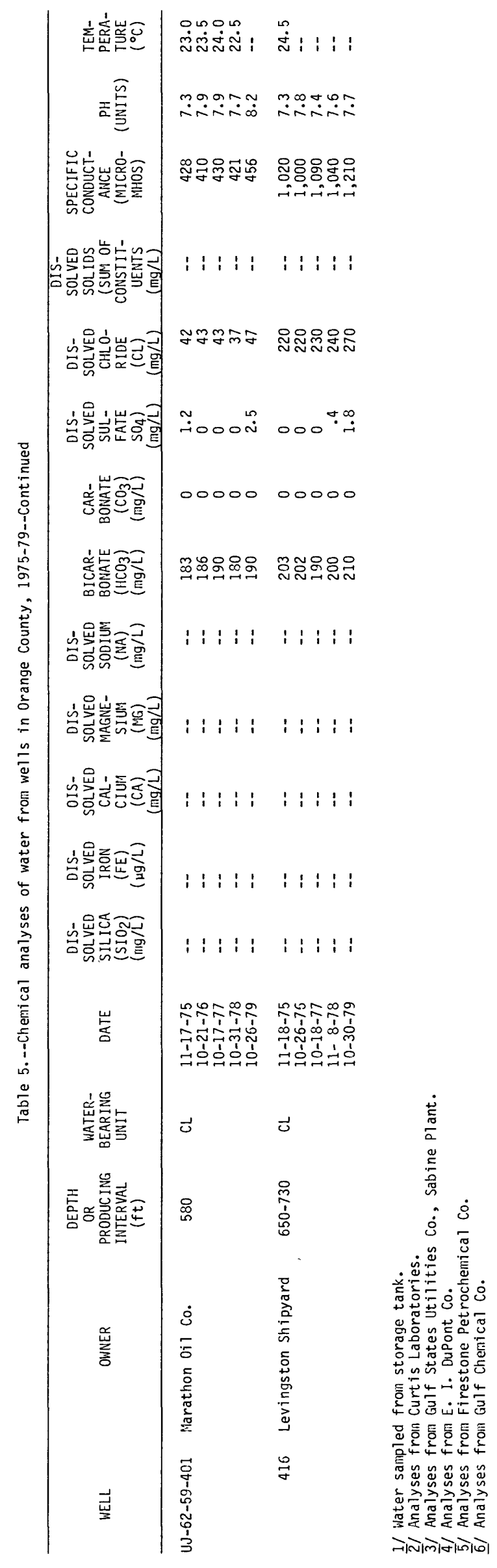

